\title{
Predicting Extinction or Explosion in a Galton-Watson Branching Process with Power Series Offspring Distribution $^{1}$
}

\author{
Peter Guttorp ${ }^{2}$ and Michael D. Perlman ${ }^{3}$ \\ University of Washington
}

\begin{abstract}
Extinction is certain in a Galton-Watson (GW) branching process if the offspring mean $\mu \leq 1$, whereas explosion is possible but not certain if $\mu>1$. Discriminating between these two possibilities is a well-studied hypothesistesting problem. However, deciding whether extinction or explosion will occur for the current realization of the process is a prediction problem. This can be formulated as a different testing problem by considering the conditional distributions of the process given extinction and explosion respectively. For power series offspring distributions, fixed-sample and sequential parametric tests are presented for the prediction problem and illustrated with data on the spread of epidemics and the populations of endangered species.
\end{abstract}

\footnotetext{
${ }^{1}$ Key words and phrases: Galton-Watson branching process; extinction; explosion; subcritical; supercritical; stochastic ordering; prediction; hypothesis testing; least favorable distribution; sequential probability ratio test; epidemic; endangered species.

${ }^{2}$ peter@stat.washington.edu. Research supported in part by National Science Foundation Grant DMS-1106862.

${ }^{3}$ michael@stat.washington.edu. Research supported in part by U.S. Department of Defense Grant H98230-10-C-0263/0000 P0004.
} 


\section{Introduction: the 2012 pertussis outbreak in Washington State}

In 2011 the weekly numbers of new pertussis (whooping cough) cases in Washington State remained fairly constant, but in 2012 the numbers increased rapidly (Figure 1, CDC (2012)). Faced with the possibility of a pandemic, the governor declared a state-wide health emergency in Week 14 and an inoculation/quarantine program was begun.

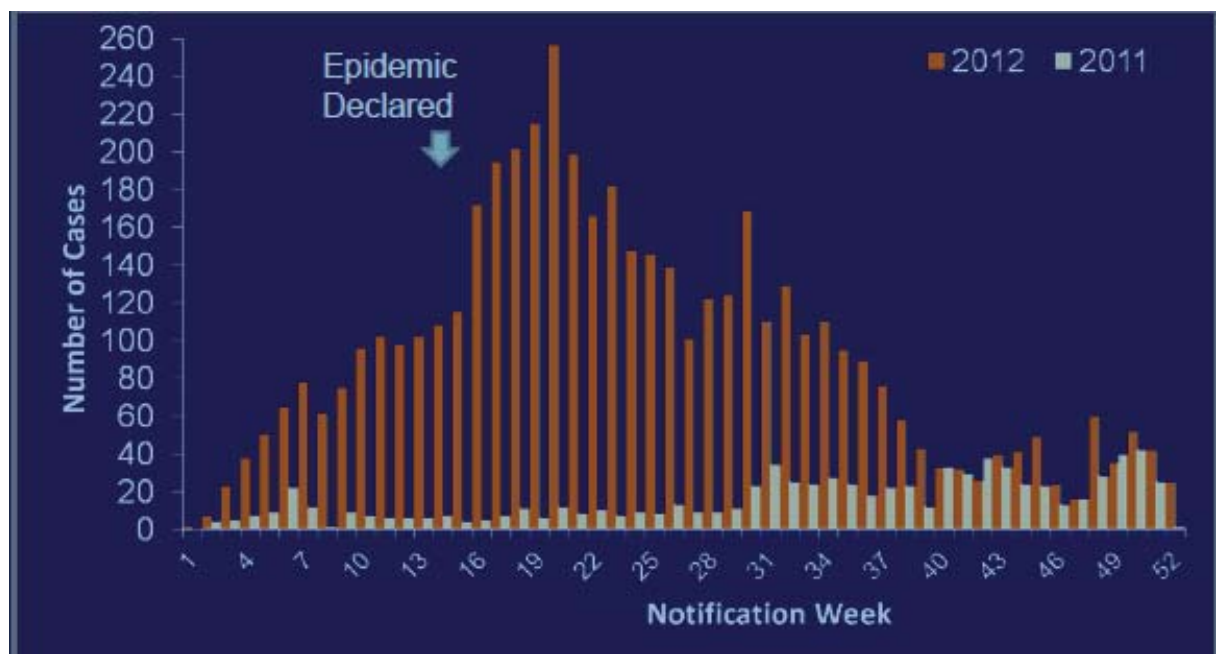

Figure 1: Weekly counts of new pertussis cases in Washington state.

The spread of an epidemic, at least in its initial stages, can be modeled as a classical Galton-Watson (GW) branching process, cf. $\S 2$. The question of predicting extinction or explosion is commonly formulated as that of testing $\mu \leq 1$ (subcriticality/criticality) vs. $\mu>1$ (supercriticality), where $\mu$ denotes the mean number of infected offspring per individual case - cf. Becker (1974), Heyde (1979), Scott (1987). ${ }^{4}$ Guttorp and Perlman (2015) use a decisiontheoretic analysis to show, however, that this problem is more complex than previous literature suggests and that the basis of a standard test procedure is somewhat dubious.

Fortunately, this testing problem usually is not the one of actual interest, because a supercritical process still may terminate with positive probability.

\footnotetext{
${ }^{4}$ Basawa and Scott (1976) and Sweeting (1978) treat a related testing problem for the supercritical case.
} 
Of more interest is the problem of predicting whether the current realization of a non-terminated process will terminate or explode.

In $§ 5-6$ this prediction problem is formulated as a different hypothesistesting problem based on the conditional distributions of the process given eventual extinction and explosion respectively. Unlike the original testing problem, this prediction problem often has relatively simple solutions in the fixed-sample $(\S 5)$ and sequential sample $(\S 6)$ cases, the latter based on the classical Wald sequential probability ratio test (SPRT), see $\S 6$. Using this procedure, explosion might have been predicted for the 2012 pertussis outbreak as early as Week 3; see Example 7.2.

Like the authors noted above who treated the original testing problem, we assume a parametric model for the offspring distribution, a power series offspring distribution (psod); see $\S 3$. The conditional distributions of a GW process given (eventual) extinction or explosion are given in $\S 2$, then specialized in $\S 3$ to the psod case. If the psod satisfies two total positivity conditions, these conditional distributions possess the stochastic monotonicity properties needed to justify our fixed- $n$ and sequential prediction methods; see $\S 4$. Yaglom's (1947) well-known exponential approximation for the distribution of the population size is extended and sharpened in $\S 5.3$ and $\S 5.4$.

\section{Conditional processes derived from a GW branching process}

The Galton-Watson branching process is a discrete-time Markov chain that describes the growth or decline of a population that reproduces by simple branching, or splitting. Applications include nuclear chain reactions, epidemics, and the population size of endangered species. The classic reference is Harris (1963, Ch. I); also see Karlin (1966), Feller (1968), Athreya and Ney (1972), Jagers (1975), Taylor and Karlin (1984), Guttorp (1991).

For each $n=0,1,2 \ldots$ let $X_{n}$ denote the population size at generation $n$; assume that $X_{0}=x_{0} \geq 1$ is known. At generation $n=0$ the $i$ th individual is replaced by a random number $\xi_{i}^{(1)} \stackrel{d}{=} \xi$ of first-generation offspring, where the offspring random variable $(\mathrm{rv}) \xi \equiv \xi_{\mathbf{p}}$ has probability distribution $\mathbf{p} \equiv\left(p_{0}, p_{1}, p_{2}, \ldots\right)$ on $\{0,1,2, \ldots\}$. The $i$-th individual in generation

$n-1$ similarly is replaced by a random number $\xi_{i}^{(n)} \stackrel{d}{=} \xi$ of $n$-th generation offspring independently of its siblings. Thus the population size in the $n$-th generation satisfies

$$
X_{n}=\xi_{1}^{(n)}+\cdots+\xi_{X_{n-1}}^{(n)}, \quad n \geq 1
$$


where $\xi_{1}^{(n)}, \ldots, \xi_{X_{n-1}}^{(n)}$ are iid rvs, each $\stackrel{d}{=} \xi$. We assume that each $p_{k}<1$ so the process is not deterministic, and that $p_{0}>0$ so extinction is possible.

Denote the probability generating function (pgf) of the offspring distribution by

$$
\phi(s) \equiv \phi_{\mathbf{p}}(s)=\mathrm{E}_{\mathbf{p}}\left(s^{\xi}\right)=\sum_{k=0}^{\infty} p_{k} s^{k}, \quad s \geq 0,
$$

and let $1 \leq \rho \equiv \rho_{\mathbf{p}} \leq \infty$ be its radius of convergence. Note that $\phi(1)=1$. Because $\phi(s)$ is convex and $p_{1}<1$, the equation

$$
\phi(s)=s
$$

has either one finite root or two distinct finite roots in $(0, \rho]$, one of which must be 1 . If (3) has one finite root in $(0, \rho]$ denote it by $u \equiv u_{\mathbf{p}}$; if $(3)$ has two distinct finite roots in $(0, \rho]$ denote them by $u \equiv u_{\mathbf{p}}$ and $v \equiv v_{\mathbf{p}}$, where $0<u<v \leq \rho$.

If $x_{0}=1$, the pgf of $X_{n}$ is the $n$-th functional iterate of $\phi$, denoted by $\phi_{n}$. For $x_{0} \geq 1$ the pgf of $X_{n}$ is $\phi_{n}^{x_{0}} \equiv\left(\phi_{n}\right)^{x_{0}}$. Either extinction $\left(X_{n}=0\right.$ for some $n \geq 1)$ or explosion $\left(X_{n} \rightarrow \infty\right)$ must occur; their probabilities are $u^{x_{0}}$ and $1-u^{x_{0}}$ respectively.

Denote the mean of the offspring distribution by $\mu \equiv \mu_{\mathbf{p}}=\mathrm{E}(\xi)$; then $\mu=\phi^{\prime}(1)$. The $\mathrm{GW}$ process $\mathbf{X} \equiv \mathbf{X}_{\mathbf{p}}$ and its pgf $\phi \equiv \phi_{\mathbf{p}}$ are called subcritical (resp., critical, supercritical) if $\mu<1(\mu=1, \mu>1)$; see Figure 2. In the subcritical case, $u=1$ and $v$ may or may not exist, see $\S 2$. In the critical case, $u=1$ and $v$ does not exist. In the supercritical case $0<u<v=1$, so both extinction and explosion occur with positive probability.

For a subcritical GW process, if $v$ exists then $\mathbf{p}, \mathbf{X}$, and $\phi$ are called extendable; in this case $1=u<v \leq \rho$ (see Figure 2). If $\rho=1$ then $v>1$ cannot exist so $\phi$ is not extendable, while if $\rho=\infty$ then $\phi$ is extendable since it grows at a quadratic rate or faster hence eventually crosses the $45^{\circ}$ line a second time beyond $1=u$. If $1<\rho<\infty$ then $\phi$ is extendable iff $\phi(\rho) \geq \rho$.

Definition 2.1. For a supercritical $G W$ process $\mathbf{X}$, define the conditional processes

$$
\begin{aligned}
\dot{\mathbf{X}} & \equiv \mathbf{X} \mid \text { extinction } \\
\ddot{\mathbf{X}} & \equiv \mathbf{X} \mid \text { explosion }
\end{aligned}
$$

If $\mathbf{X}$ is subcritical or critical, define $\dot{\mathbf{X}}=\mathbf{X}$. 
Supercritical

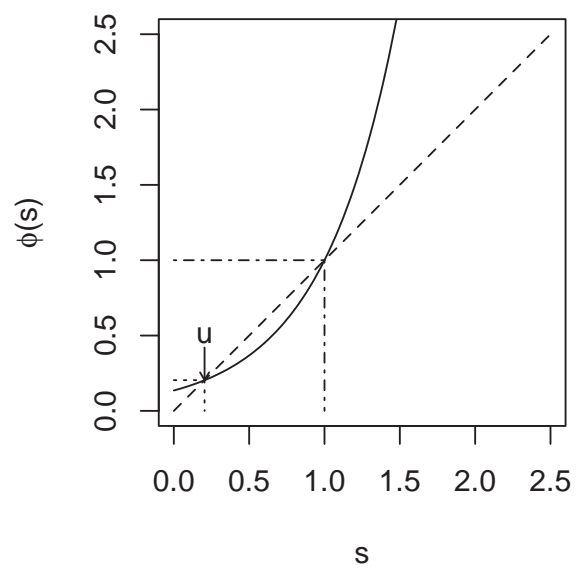

Subcritical, extendable

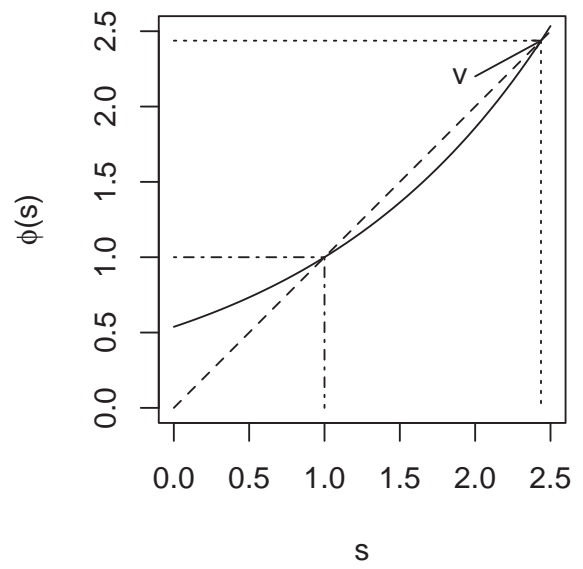

Figure 2: The duality between supercritical and extendable subcritical pgfs.

Proposition 2.1. The set of supercritical $G W$ processes conditional on extinction coincides with the set of subcritical extendable $G W$ processes.

Proof. If $\mathbf{X}$ is supercritical it is well known ${ }^{5}$ that $\dot{\mathbf{X}}$ is a subcritical GW process with offspring pgf

$$
\dot{\phi}(s)=\frac{\phi(u s)}{u}
$$

and offspring mean $\dot{\mu}=\phi^{\prime}(u)<1$. Furthermore $\dot{\phi}$ is extendable with second root $\dot{v}=1 / u$.

Now suppose that $\mathbf{X}$ is subcritical and extendable. Define

$$
\tilde{\phi}(s)=\frac{\phi(v s)}{v} .
$$

It is straightforward to verify that $\tilde{\phi}$ is a supercritical offspring pgf with offspring mean $\tilde{\mu}=\phi^{\prime}(v)>1$ and extinction probability $\tilde{u}=1 / v$. Denote the corresponding supercritical GW process by $\tilde{\mathbf{X}}$. Then

$$
\dot{\tilde{\phi}}(s)=\frac{\phi(\tilde{u} v s)}{\tilde{u} v}=\phi(s) .
$$

\footnotetext{
${ }^{5}$ Waugh (1958, p.248), Athreya, Ney (1972, §I.12, Theorem 3), Guttorp (1991, p.101) .
} 
Futhermore, if $\mathbf{X}$ is supercritical then

$$
\tilde{\dot{\phi}}(s)=\frac{\phi(u \dot{v} s)}{u \dot{v}}=\phi(s) .
$$

This establishes the asserted result.

Successive conditioning on $X_{1}, \ldots, X_{n-1}$ in (1) shows that the joint probability mass function (pmf) $f \equiv f_{\mathbf{p}}$ of $\mathbf{X}_{n} \equiv\left(X_{1}, \ldots, X_{n}\right)$ is given by

$$
f\left(\mathbf{x}_{n}\right) \equiv \operatorname{Pr}_{\mathbf{p}}\left[\mathbf{X}_{n}=\mathbf{x}_{n}\right]=\prod_{i=1}^{n} h_{\mathbf{p}}\left(x_{i-1}, x_{i}\right) \equiv h_{\mathbf{p}}\left(\mathbf{x}_{n}\right)
$$

(e.g. Jagers (1975, eqn. (2.1.2)), where

$$
h_{\mathbf{p}}(k, l)=\sum_{r_{1}+\cdots+r_{k}=l} p_{r_{1}} \cdots p_{r_{k}}
$$

Note that $h_{\mathbf{p}}(k, l)$ is the coefficient of $s^{l}$ in the power series $\left[\phi_{\mathbf{p}}(s)\right]^{k}$.

From Bayes' formula, the pmf of $\dot{\mathbf{X}}_{n} \equiv\left(\dot{X}_{1}, \ldots, \dot{X}_{n}\right)$ is given by

$$
\begin{aligned}
\dot{f}\left(\mathbf{x}_{n}\right) \equiv \dot{f}_{\mathbf{p}}\left(\mathbf{x}_{n}\right) & =\operatorname{Pr}_{\mathbf{p}}\left[\mathbf{X}_{n}=\mathbf{x}_{n} \mid \text { extinction }\right] \\
& =\frac{\operatorname{Pr}\left[\text { extinction } \mid \mathbf{X}_{n}=\mathbf{x}_{n}\right] \operatorname{Pr}\left[\mathbf{X}_{n}=\mathbf{x}_{n}\right]}{\operatorname{Pr}[\text { extinction }]} \\
& =u^{x_{n}-x_{0}} f\left(\mathbf{x}_{n}\right) .
\end{aligned}
$$

Similarly the pmf of $\ddot{\mathbf{X}}_{n} \equiv\left(\ddot{X}_{1}, \ldots, \ddot{X}_{n}\right)$ is given by

$$
\ddot{f}\left(\mathbf{x}_{n}\right) \equiv \ddot{f}_{\mathbf{p}}\left(\mathbf{x}_{n}\right)=\left(\frac{1-u^{x_{n}}}{1-u^{x_{0}}}\right) f\left(\mathbf{x}_{n}\right), \quad \mathbf{x}_{n}>0,
$$

where $\mathbf{x}_{n}>0$ means that $x_{1}>0, \ldots, x_{n}>0$. From (12) and (13), $\dot{\mathbf{X}}$ and $\ddot{\mathbf{X}}$ are Markovian with transition probabilities

$$
\begin{aligned}
& \dot{f}\left(x_{n} \mid x_{n-1}\right)=u^{x_{n}-x_{n-1}} h_{\mathbf{p}}\left(x_{n-1}, x_{n}\right), \\
& \ddot{f}\left(x_{n} \mid x_{n-1}\right)=\left(\frac{1-u^{x_{n}}}{1-u^{x_{n-1}}}\right) h_{\mathbf{p}}\left(x_{n-1}, x_{n}\right), \quad x_{n-1}, x_{n}>0,
\end{aligned}
$$

respectively. However, $\ddot{\mathrm{X}}$ is not a GW process because some individuals may die without offspring even though explosion occurs. 


\section{The GW process with power series offspring distribution}

Following Becker (1974) we now specialize this discussion to a parametric model for the offspring distribution $\mathbf{p} \equiv\left(p_{0}, p_{1}, \ldots\right)$. The power series offspring distribution (psod) $\mathbf{p}_{\theta} \equiv\left(p_{\theta ; 0}, p_{\theta ; 1}, \ldots\right)$ is given by

$$
p_{\theta ; k}=\frac{a_{k} \theta^{k}}{A(\theta)}, \quad k=0,1, \ldots, \quad 0<\theta<\psi
$$

where $\left(a_{0}, a_{1}, \ldots\right) \equiv \mathbf{a}$ are nonnegative constants, $\theta$ is the unknown parameter, $A(\theta)=\sum a_{k} \theta^{k}$, and $0<\psi \leq \infty$ is the radius of convergence of $A(\cdot)$. We assume that $a_{0}>0$ so extinction is possible, and that $a_{k}>0$ for at least one $k \geq 2$ so growth is possible; without loss of generality we may take $a_{0}=1$. For simplicity of exposition we limit attention to the case where $A\left(\psi_{-}\right)=\infty$; this includes the familiar Poisson, binomial, geometric, negative binomial, binary splitting, and logarithmic series distributions.

Denote $\mathbf{X}_{\mathbf{p}_{\theta}}, \xi_{\mathbf{p}_{\theta}}, f_{\mathbf{p}_{\theta}}, \phi_{\mathbf{p}_{\theta}}, \rho_{\mathbf{p}_{\theta}}, u_{\mathbf{p}_{\theta}}, v_{\mathbf{p}_{\theta}}, \mu_{\mathbf{p}_{\theta}}$ by $\mathbf{X}_{\theta}, \xi_{\theta}, f_{\theta}, \phi_{\theta}, \rho_{\theta}, u_{\theta}, v_{\theta}$, $\mu_{\theta}$ respectively. By (2) and (16), $\phi_{\theta}$ has radius of convergence $\rho_{\theta}=\psi / \theta$ and

$$
\phi_{\theta}(s)=\frac{A(\theta s)}{A(\theta)}=\frac{B(\theta s)}{B(\theta)} s, \quad 0<s<\rho_{\theta},
$$

where $B(\theta)=A(\theta) / \theta$ (see Figure 3 ). Here $B(\theta)$ is a strictly convex positive function on $(0, \psi)$ with $B(0+)=B(\psi-)=\infty$, so $B(\cdot)$ has a unique minimum at some $\tau \in(0, \psi)$ with $B^{\prime}(\tau)=0 ; B(\theta)$ is strictly decreasing for $\theta<\tau$ and strictly increasing for $\theta>\tau$.

It follows from (17) that for $\theta \in(0, \psi)$,

$$
\begin{aligned}
\mathrm{E}_{\theta}(\xi) \equiv \mu_{\theta} & =\frac{\theta A^{\prime}(\theta)}{A(\theta)} \\
\frac{\mu_{\theta}-1}{\theta} & =\frac{B^{\prime}(\theta)}{B(\theta)}=\frac{d \log B(\theta)}{d \theta}, \\
\operatorname{Var}_{\theta}(\xi) \equiv \sigma_{\theta}^{2} & =\theta \frac{d \mu_{\theta}}{d \theta} .
\end{aligned}
$$

By $(19), \mu_{\tau}=1$ so $\mathbf{X}_{\tau}$ is critical. By (20), $\mu_{\theta}$ is strictly increasing in $\theta$, hence the subcritical and supercritical parameter spaces are both open intervals:

$$
\begin{aligned}
& \left\{\theta \mid \mu_{\theta}<1\right\}=(0, \tau) \quad \text { (subcritical) } \\
& \left\{\theta \mid \mu_{\theta}>1\right\}=(\tau, \psi) \quad \text { (supercritical). }
\end{aligned}
$$




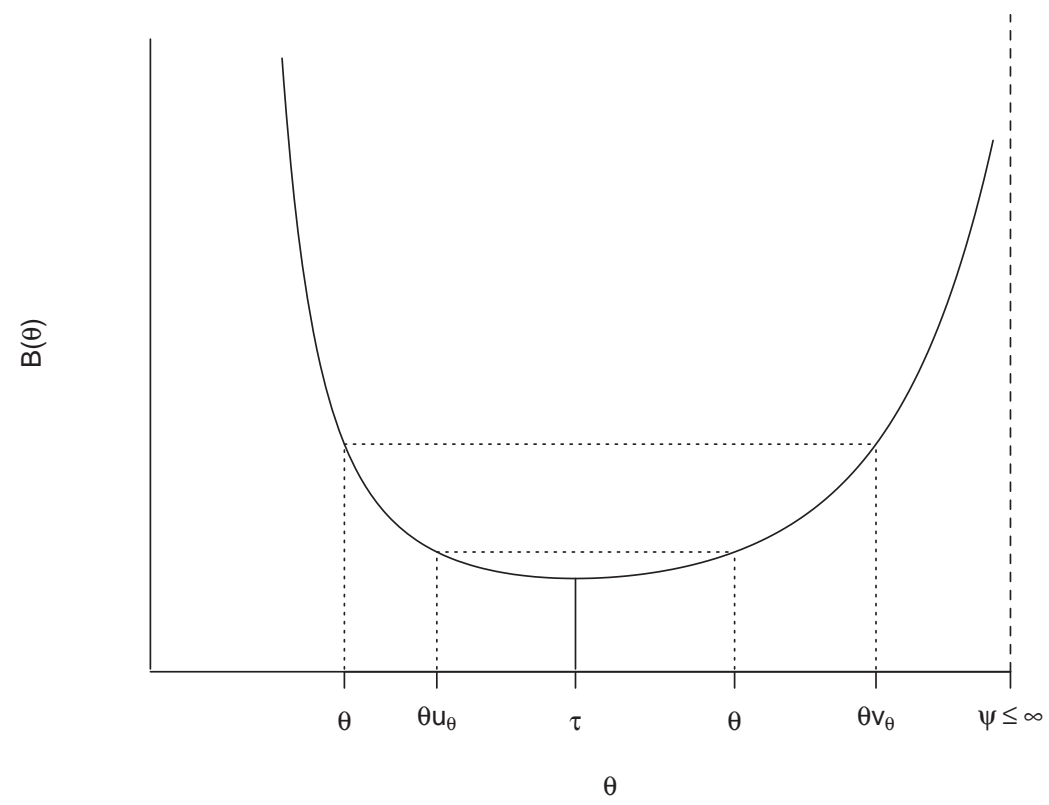

Figure 3: The function $B(\theta)=A(\theta) / \theta$.

If $\theta \in(\tau, \psi)$ then from (3) and (17), $u_{\theta}$ is the unique solution to

$$
B\left(\theta u_{\theta}\right)=B(\theta), \quad 0<\theta u_{\theta}<\tau .
$$

If $\theta \in(0, \tau)$ then $v_{\theta}$ is the unique solution to

$$
B(\theta)=B\left(\theta v_{\theta}\right), \quad \tau<\theta v_{\theta}<\psi .
$$

Thus each subcritical $\mathbf{X}_{\theta}$ is extendable. It follows from the uniqueness of the solutions of (23) and (24) that

$$
\begin{array}{ll}
v_{\theta u_{\theta}}=u_{\theta}^{-1} & \text { for } \theta \in(\tau, \psi), \\
u_{\theta v_{\theta}}=v_{\theta}^{-1} & \text { for } \theta \in(0, \tau) .
\end{array}
$$

Proposition 3.1. (i) For $\theta \in(\tau, \psi), \theta u_{\theta}$ strictly decreases from $\tau$ to 0 ; $u_{\theta}$ strictly decreases from 1 to 0.

(ii) For $\theta \in(0, \tau), \theta v_{\theta}$ strictly decreases from $\psi$ to $\tau$; $v_{\theta}$ strictly decreases from $\infty$ to 1 .

Proof. (i) It follows from (23) and (19) that for $\theta \in(\tau, \psi)$,

$$
d_{\theta} \equiv \frac{d\left(\theta u_{\theta}\right)}{d \theta}=\left(\frac{\mu_{\theta}-1}{\mu_{\theta u_{\theta}}-1}\right) u_{\theta} .
$$


Thus $d_{\theta}<0$ because $\theta u_{\theta}<\tau<\theta$, so $\theta u_{\theta}$ is strictly decreasing, a fortiori $u_{\theta}$ is strictly decreasing. As $\theta \downarrow \tau, B(\theta) \downarrow B(\tau)$, its unique minimum, hence $\theta u_{\theta} \uparrow \tau$ by (23), so $u_{\theta} \uparrow 1$. As $\theta \uparrow \psi, B(\theta) \uparrow \infty$, hence $\theta u_{\theta} \downarrow 0$ by (23), so $u_{\theta} \downarrow 0$.

(ii) It follows from (24) and (19) that for $\theta \in(0, \tau)$,

$$
\frac{d\left(\theta v_{\theta}\right)}{d \theta}=\left(\frac{\mu_{\theta}-1}{\mu_{\theta v_{\theta}}-1}\right) v_{\theta}
$$

which is $<0$ because $\tau<\theta v_{\theta}<\psi$. The remaining results are verified as in (i). Alternatively, (25) and (26) can be applied to obtain $v_{\tau-}$ and $v_{0+}$.

Proposition 3.1, (25), and (26) establish analytically a 1-1 relation between the subcritical $(0, \tau)$ and supercritical $(\tau, \psi)$ parameter spaces. The corresponding probabilistic relation between the subcritical and supercritical processes themselves is now presented.

Proposition 3.2. The set of supercritical processes $\left\{\dot{\mathbf{X}}_{\theta} \mid \theta \in(\tau, \psi)\right\}$ conditional on extinction coincides with the set of subcritical processes $\left\{\mathbf{X}_{\theta} \mid \theta \in\right.$ $(0, \tau)\}$. Specifically,

$$
\begin{array}{ll}
\dot{\mathbf{X}}_{\theta} \stackrel{d}{=} \mathbf{X}_{\theta u_{\theta}}, & \theta \in(\tau, \psi), \\
\mathbf{X}_{\theta} \stackrel{d}{=} \dot{\mathbf{X}}_{\theta v_{\theta}}, & \theta \in(0, \tau) .
\end{array}
$$

(Note too that $\dot{\mathbf{X}}_{\tau} \stackrel{d}{=} \mathbf{X}_{\tau}$.)

Proof. Suppose first that $\mathbf{X}_{\theta}$ is supercritical, i.e., $\theta \in(\tau, \psi)$. From $(6), \dot{\mathbf{X}}_{\theta}$ is a subcritical GW process with offspring pgf in the same psod family (16):

$$
\dot{\phi}_{\theta}(s)=\frac{A\left(\theta u_{\theta} s\right)}{A(\theta) u_{\theta}}=\frac{A\left(\theta u_{\theta} s\right)}{A\left(\theta u_{\theta}\right)}=\phi_{\theta u_{\theta}}(s) ;
$$

cf. Becker (1974, p.394). Since $\theta u_{\theta}<\tau, \mathbf{X}_{\theta u_{\theta}}$ is subcritical.

Suppose next that $\mathbf{X}_{\theta}$ is subcritical, i.e., $\theta \in(0, \tau)$. A similar argument using (7) shows that $\tilde{\mathbf{X}}_{\theta}$ is a supercritical GW process with offspring pgf

$$
\tilde{\phi}_{\theta}(s)=\phi_{\theta v_{\theta}}(s) \text {. }
$$

Now apply (8) to obtain $\phi_{\theta}=\dot{\phi}_{\theta v_{\theta}}$; since $\theta v_{\theta}>\tau, \mathbf{X}_{\theta v_{\theta}}$ is supercritical. 
Example 3.1: the Poisson $(\theta)$ psod. Here $p_{k ; \theta}=e^{-\theta} \theta^{k} / k !, 0<\theta<\infty$, so $a_{k}=1 / k !, A(\theta)=e^{\theta}, \psi=\infty, A(\psi-)=\infty, B(\theta)=e^{\theta} / \theta$, and $\phi_{\theta}(s)=e^{\theta(s-1)}$. Then $\mu_{\theta}=\sigma_{\theta}^{2}=\theta, \tau=1$, and $u_{\theta}$ and $v_{\theta}$ satisfy the equation

$$
e^{\theta(s-1)}=s
$$

by (23) and (24). This cannot be solved explicitly, but necessarily

$$
\begin{cases}u_{\theta}=1, v_{\theta}>1 & \text { if } \theta<1 \text { (subcritical) } \\ u_{\theta}<1, v_{\theta}=1 & \text { if } \theta>1 \text { (supercritical) }\end{cases}
$$

Example 3.2: the negative binomial $\mathbf{N B}(r, \theta)$ and geometric $\operatorname{GM}(\theta)$ psods. For fixed $r>0$, the $\mathrm{NB}(r, \theta)$ psod has $p_{k ; \theta}=\frac{\Gamma(r+k)}{\Gamma(r) k !}(1-\theta)^{r} \theta^{k}$, $0<\theta<1$. Here $a_{k}=\frac{\Gamma(r+k)}{\Gamma(r) k !}, A(\theta)=\frac{1}{(1-\theta)^{r}}, \psi=1, B(\theta)=\frac{1}{\left[\theta(1-\theta)^{r}\right]}$, and $\phi_{\theta}(s)=\frac{(1-\theta)^{r}}{(1-\theta s)^{r}}$. Also $\mu_{\theta}=\frac{r \theta}{1-\theta}, \sigma_{\theta}^{2}=\frac{r \theta}{(1-\theta)^{2}}, \tau=\frac{1}{1+r}$, and $u_{\theta}$ and $v_{\theta}$ satisfy the equation

$$
(1-\theta)^{r}=(1-\theta s)^{r} s
$$

This can be solved explicitly for the $\operatorname{GM}(\theta) \equiv \mathrm{NB}(1, \theta)$ psod where $r=1$ and $\tau=1 / 2$ :

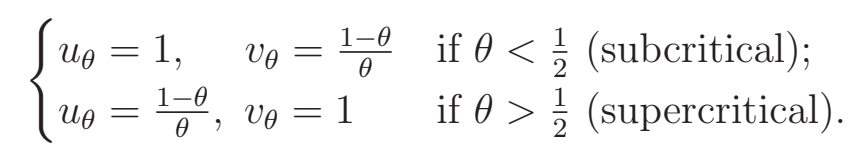

Here the relations (25) and (26) can be verified directly.

Example 3.3: binary splitting. Take $a_{0}=a_{2}=1$ and $a_{k}=0$ for $k \neq 2$. Thus $A(\theta)=1+\theta^{2}$ for $0<\theta<\infty \equiv \psi$, so $p_{0 ; \theta}=\frac{1}{1+\theta^{2}}, p_{2 ; \theta}=\frac{\theta^{2}}{1+\theta^{2}}$, and $p_{k ; \theta}=0$ for $k \neq 0,2$. Here $B(\theta)=\theta^{-1}+\theta, \phi_{\theta}(s)=\frac{1+\theta^{2} s^{2}}{1+\theta^{2}}, \mu_{\theta}=\frac{2 \theta^{2}}{1+\theta^{2}}$, $\sigma_{\theta}^{2}=\frac{4 \theta^{2}}{\left(1+\theta^{2}\right)^{2}}, \tau=1$, and

$$
\begin{cases}u_{\theta}=1, \quad v_{\theta}=\frac{1}{\theta^{2}} & \text { if } \theta<1 \text { (subcritical) } \\ u_{\theta}=\frac{1}{\theta^{2}}, \quad v_{\theta}=1 & \text { if } \theta>1 \text { (supercritical). }\end{cases}
$$

Again the relations (25) and (26) can be verified directly.

\section{Stochastic orderings for a psod GW process}

Let $W, Z, \mathbf{W}_{n} \equiv\left(W_{1}, \ldots, W_{n}\right), \mathbf{Z}_{n} \equiv\left(Z_{1}, \ldots, Z_{n}\right)$, and $\mathbf{W} \equiv\left(W_{1}, \ldots\right), \mathbf{Z} \equiv$ $\left(Z_{1}, \ldots\right)$ be nonnegative-integer-valued random variables, random vectors, 
and discrete-time stochastic processes, respectively. We say $W$ is stochastically smaller than $Z$, written $W \prec Z$, if $\mathrm{E}[g(W)] \leq \mathrm{E}[g(Z)]$ for all increasing bounded nonnegative functions $g$ on the nonnegative integers $\mathbb{Z}_{0}$ with strict inequality for at least one $g$. It is straightforward to show that if $U, V, W, Z$ are independent, then

$$
U \prec V \text { and } W \prec Z \Longrightarrow U+W \prec V+Z \text {. }
$$

Similarly, we write $\mathbf{W}_{n} \prec \mathbf{Z}_{n}$ if

$$
\mathrm{E}\left[g\left(\mathbf{W}_{n}\right)\right] \leq \mathrm{E}\left[g\left(\mathbf{Z}_{n}\right)\right]
$$

for all increasing bounded nonnegative functions $g$ on $\mathbb{Z}_{0}^{n}$ with strict inequality for at least one $g$. Finally, we write $\mathbf{W} \prec \mathbf{Z}$ if $\mathbf{W}_{n} \prec \mathbf{Z}_{n}$ for all $n=1,2, \ldots$. The next lemma follows directly from (1) and (38).

Lemma 4.1. Let $\mathbf{X}$ and $\mathbf{X}^{\prime}$ be $G W$ processes with offspring rv's $\xi$ and $\xi^{\prime}$ respectively. If $\xi \prec \xi^{\prime}$ then $\mathbf{X} \prec \mathbf{X}^{\prime}$.

Stochastic orderings satisfied by a GW process $\mathbf{X}_{\theta}$ with psod (16) and by the conditional processes $\dot{\mathbf{X}}_{\theta}$ and $\ddot{\mathbf{X}}_{\theta}$ are now developed. These will be useful for the testing and prediction problems treated below.

From (10), (11), and (16), the pmf of $\left(\mathbf{X}_{\theta}\right)_{n}$ is

$$
f_{\theta}\left(\mathbf{x}_{n}\right)=\frac{\theta^{y_{n}-x_{0}}}{(A(\theta))^{y_{n-1}}} h_{\mathbf{a}}\left(\mathbf{x}_{n}\right), \quad \mathbf{x}_{n} \in R_{\mathbf{a}, n}^{\prime},
$$

where $y_{n}=x_{0}+x_{1}+\cdots+x_{n}$ and $R_{\mathbf{a}, n}^{\prime}=\left\{\mathbf{x}_{n} \mid h_{\mathbf{a}}\left(\mathbf{x}_{n}\right)>0\right\}$. Then (12), (13), and (40) give the following:

$$
\begin{aligned}
\text { for } \theta>0: & \dot{f}_{\theta}\left(\mathbf{x}_{n}\right)=u_{\theta}^{x_{n}-x_{0}} \frac{\theta^{y_{n}-x_{0}}}{(A(\theta))^{y_{n-1}}} h_{\mathbf{a}}\left(\mathbf{x}_{n}\right), \\
\text { for } \theta>\tau: & \ddot{f}_{\theta}\left(\mathbf{x}_{n}\right)=\left(\frac{1-u_{\theta}^{x_{n}}}{1-u_{\theta}^{x_{0}}}\right) \frac{\theta^{y_{n}-x_{0}}}{(A(\theta))^{y_{n-1}}} h_{\mathbf{a}}\left(\mathbf{x}_{n}\right), \quad \mathbf{x}_{n}>0 .
\end{aligned}
$$

The transition probabilities are obtained from (40)-(42) (recall (14)-(15)):

$$
\begin{aligned}
& f_{\theta}\left(x_{n} \mid x_{n-1}\right)=\frac{\theta^{x_{n}}}{(A(\theta))^{x_{n-1}}} h_{\mathbf{a}}\left(x_{n-1}, x_{n}\right) \\
& \dot{f}_{\theta}\left(x_{n} \mid x_{n-1}\right)=u_{\theta}^{x_{n}-x_{n-1}} \frac{\theta^{x_{n}}}{(A(\theta))^{x_{n-1}}} h_{\mathbf{a}}\left(x_{n-1}, x_{n}\right) \\
& \ddot{f}_{\theta}\left(x_{n} \mid x_{n-1}\right)=\left(\frac{1-u_{\theta}^{x_{n}}}{1-u_{\theta}^{x_{n-1}}}\right) \frac{\theta^{x_{n}}}{(A(\theta))^{x_{n-1}}} h_{\mathbf{a}}\left(x_{n-1}, x_{n}\right), \quad x_{n-1}, x_{n}>0
\end{aligned}
$$


The definitions of $\ddot{f}_{\theta}(\cdot)$ and $\ddot{f}_{\theta}(\cdot \mid \cdot)$ can be extended to the critical case $\theta=\tau$ :

$$
\begin{aligned}
\ddot{f}_{\tau}\left(\mathbf{x}_{n}\right) & =\lim _{\theta \downarrow \tau} \ddot{f}_{\theta}\left(\mathbf{x}_{n}\right) \\
& =\frac{x_{n}}{x_{0}} \frac{\tau^{y_{n}-x_{0}}}{(A(\tau))^{y_{n-1}}} h_{\mathbf{a}}\left(\mathbf{x}_{n}\right), \quad \mathbf{x}_{n}>0 ; \\
\ddot{f}_{\tau}\left(x_{n} \mid x_{n-1}\right) & =\frac{x_{n}}{x_{n-1}} \frac{\tau^{x_{n}}}{(A(\tau))^{x_{n-1}}} h_{\mathbf{a}}\left(x_{n-1}, x_{n}\right), \quad x_{n-1}, x_{n}>0 .
\end{aligned}
$$

Denote the resulting Markov process by $\ddot{\mathbf{X}}_{\tau} \cdot{ }^{6}$ By (46) and Scheffe's Theorem,

$$
\ddot{\mathbf{X}}_{\theta} \stackrel{L_{1}}{\longrightarrow} \ddot{\mathbf{X}}_{\tau} \quad \text { as } \theta \downarrow \tau \text {. }
$$

Proposition 4.1. (i) $\mathbf{X}_{\theta}$ is stochastically increasing for $\theta \in(0, \psi)$, that is, $\theta<\theta^{\prime} \Rightarrow \mathbf{X}_{\theta} \prec \mathbf{X}_{\theta^{\prime}}$.

(ii) $\dot{\mathbf{X}}_{\theta}$ is stochastically decreasing for $\theta \in[\tau, \psi)$, that is, $\theta<\theta^{\prime} \Rightarrow \dot{\mathbf{X}}_{\theta} \succ \dot{\mathbf{X}}_{\theta^{\prime}}$.

Proof. (i) follows from Lemma 4.1 since $\theta<\theta^{\prime} \Rightarrow \xi_{\theta} \prec \xi_{\theta^{\prime}}$ by the strict monotone likelihood ratio (MLR) property of the psod family. ${ }^{7}$ (ii) follows from (i), (29), and Proposition 3.1(i).

The verifications of the stochastic orderings of $\mathbf{X}_{\theta}$ and $\dot{\mathbf{X}}_{\theta}$ are straightforward because these are GW processes. However, $\ddot{\mathbf{X}}_{\theta}$ is not a GW process so its stochastic ordering properties if any are not apparent. Although it might appear that $\ddot{\mathbf{X}}_{\theta}$ should inherit the stochastic increasing property of $\mathbf{X}_{\theta}$, upon closer examination this is not obvious. Conditional on ultimate explosion, as $\theta$ increases above the critical value $\tau$ those trajectories of $\mathbf{X}_{\theta}$ with relatively small initial values might have increasing likelihood of survival, hence for fixed $n,\left(\ddot{\mathbf{X}}_{\theta}\right)_{n}$ might tend to decrease stochastically, not increase.

In Proposition 4.2(iii) it will be shown, however, that $\ddot{\mathbf{X}}_{\theta}$ is indeed stochastically increasing for $\theta \geq \tau$ provided that two additional conditions are imposed, namely TP2a and/or TP2b (see below) based on total positivity of order 2 (TP2). Also, it is shown in Proposition 4.2(i) that under TP2a alone, the conditional random vector $\left(\mathbf{X}_{\theta}\right)_{n} \mid X_{\theta, n}>0$ is stochastically increasing for $\theta \leq \tau$.

\footnotetext{
${ }^{6}$ This is not to be interpreted as $\mathbf{X}_{\tau} \mid$ explosion, which is vacuous.

${ }^{7}$ Lehmann and Romano (2005) Lemma 3.4.2 and Problem 3.39; Karlin (1968) Proposition 3.1 and the discussion following Proposition 3.3, both in Chapter 1.
} 
Karlin (1968) is the primary reference for total positivity. The TP2 property is equivalent to MLR, cf. Lehmann and Romano (2005, Problem 50)). The following results for the TP2 and FKG properties appear in Kemperman (1977) and Perlman and Olkin (1980).

Definition 4.1. Let $f(\mathbf{x})$ be a nonnegative function defined on a measurable rectangle $\mathbf{R}=\prod_{i=1}^{n} R_{i} \subseteq \mathbb{R}^{n}$. Then $f$ satisfies the $F K G$ condition on $\mathbf{R}$ if

$$
f\left(\mathbf{x}_{n}\right) f\left(\mathbf{y}_{n}\right) \leq f\left(\mathbf{x}_{n} \wedge \mathbf{y}_{n}\right) f\left(\mathbf{x}_{n} \vee \mathbf{y}_{n}\right) \quad \forall \mathbf{x}_{n}, \mathbf{y}_{n} \in \mathbf{R},
$$

where $\mathbf{x}_{n} \wedge \mathbf{y}_{n}=\left(x_{1} \wedge y_{1}, \ldots, x_{n} \wedge y_{n}\right)$ and $\mathbf{x}_{n} \vee \mathbf{y}_{n}=\left(x_{1} \vee y_{1}, \ldots, x_{n} \vee y_{n}\right)$; we say that $f$ is FKG on $\mathbf{R}$. TP2 is defined as $F K G$ for $n=2$.

Some properties of TP2 and FKG: If $h\left(x_{i}, x_{j}\right)$ is TP2 on $R_{i} \times R_{j}$ in a single pair $\left(x_{i}, x_{j}\right)$ then $f\left(\mathbf{x}_{n}\right) \equiv h\left(x_{i}, x_{j}\right)$ is FKG on $\mathbf{R}$. If $f_{1}, \ldots, f_{m}$ are FKG on $\mathbf{R}$ then so is $\prod_{i=1}^{m} f_{i}$. If $f\left(\mathbf{x}_{n}\right)=h_{i}\left(x_{i}\right)$ for a single $i$ then $f$ is trivially FKG on $\mathbf{R}$, so $f\left(\mathbf{x}_{n}\right)=\prod_{i=1}^{n} h_{i}\left(x_{i}\right)$ is also trivially FKG. If $f$ is FKG on $\mathbf{R}^{*} \equiv \prod R_{i}^{*}$ and if, for each $i=1, \ldots, n, \beta_{i}: R_{i} \rightarrow R_{i}^{*}$ is increasing in $x_{i}$, then $f\left(\beta_{1}\left(x_{1}\right), \ldots, f\left(\beta_{n}\left(x_{n}\right)\right)\right.$ is $\mathrm{FKG}$ on $\mathbf{R} \equiv \prod R_{i}$.

Lemma 4.2. (The FKG Inequality). Let $\mathbf{Z}$ be a random vector with an $F K G$ pdf $f$ on $\mathbf{R}$ w.r.to a product measure $\nu$ and let $g, h$ be component-wise increasing nonnegative functions on $\mathbf{R} \cap\{f>0\}$. Then

$$
\mathrm{E}[g(\mathbf{Z}) h(\mathbf{Z})] \geq \mathrm{E}[g(\mathbf{Z})] \mathrm{E}[h(\mathbf{Z})] .
$$

Strict inequality holds in (51) if $g$ is nonconstant w.r.to $f(\operatorname{Pr}[g(Z)=c]<1$ for all constants $c$ ) and $h$ is strictly increasing.

Proof. Perlman and Olkin (1980, Propositions 2.4, 2.6, and Remark 2.5.)

Condition TP2a: $h_{\mathbf{a}}(x, y)$ is TP2 in $(x, y)$ for $x, y=1,2, \ldots$ (Note that $h_{\mathbf{a}}(x, y)$ is the coefficient of $\theta^{y}$ in the power series $[A(\theta)]^{x}$.)

Condition TP2b: $\left(1-u_{\theta}^{x}\right) \theta^{x}$ is TP2 in $(x, \theta)$ for $x=1,2, \ldots$ and $\tau<\theta<\psi$.

A sufficient condition for TP2a to hold is that $\left\{a_{k} \mid k=0,1, \ldots\right\}$ is a onesided Polya frequency sequence of order 2 (PF2); cf. Karlin (1968, (ii) on pp.142-3, also Ch.8).

Let $\left(\mathbf{X}_{\theta}\right)_{n}^{+}$denote the conditional random vector $\left(\mathbf{X}_{\theta}\right)_{n} \mid X_{\theta, n}>0$. For notational convenience the subscript $\theta$ sometimes will be omitted. The conditional pmf of $\left(\mathbf{X}_{\theta}\right)_{n}^{+} \equiv \mathbf{X}_{n}^{+}$is given by

$$
f_{\theta}^{+}\left(\mathbf{x}_{n}\right)=\operatorname{Pr}_{\theta}\left[\mathbf{X}_{n}=\mathbf{x}_{n} \mid X_{n}>0\right]=b_{\theta, n} f_{\theta}\left(\mathbf{x}_{n}\right), \quad \mathbf{x}_{n}>0,
$$


where $b_{\theta, n}^{-1}=\operatorname{Pr}_{\theta}\left[X_{n}>0\right]$. Note that $X_{n}>0 \Rightarrow \mathbf{X}_{n}>0$. Clearly $\mathbf{X}_{n}^{+} \succ \mathbf{X}_{n}$ and $\dot{\mathbf{X}}_{n}^{+} \succ \dot{\mathbf{X}}_{n}$ for all $\theta>0$, while $\ddot{\mathbf{X}}_{n}^{+} \equiv \ddot{\mathbf{X}}_{n}$ for $\theta \geq \tau$.

Proposition 4.2. (i) If TP2a holds then for each $n \geq 1,\left(\mathbf{X}_{\theta}\right)_{n}^{+}$is stochastically increasing for $\theta \in(0, \tau]$. Therefore, by Propositions 3.1 and 3.2, $\left(\dot{\mathbf{X}}_{\theta}\right)_{n}^{+} \stackrel{d}{=}\left(\mathbf{X}_{\theta u_{\theta}}\right)_{n}^{+}$is stochastically decreasing for $\theta \in[\tau, \psi)$.

(ii) If TP2a holds then for each $n \geq 1,\left(\dot{\mathbf{X}}_{\tau}\right)_{n} \prec\left(\dot{\mathbf{X}}_{\tau}\right)_{n}^{+} \prec\left(\ddot{\mathbf{X}}_{\tau}\right)_{n}^{+} \equiv\left(\ddot{\mathbf{X}}_{\tau}\right)_{n}$.

(iii) If TP2 $a$ and TP2b hold, $\ddot{\mathbf{X}}_{\theta}$ is stochastically increasing for $\theta \in[\tau, \psi)$.

Proof. (i) We will show that $\mathrm{E}_{\theta}\left[g\left(\mathbf{X}_{n}^{+}\right)\right]$is strictly increasing in $\theta \in(0, \tau]$ for any increasing bounded nonconstant $g \geq 0$ on $\mathbb{Z}_{+}^{n}$, where $\mathbb{Z}_{+}$denotes the positive integers. The FKG inequality will yield the required result as follows: for $0<\theta_{1}<\theta_{2} \leq \tau$,

$$
\begin{aligned}
\mathrm{E}_{\theta_{2}}\left[g\left(\mathbf{X}_{n}^{+}\right)\right] & =\mathrm{E}_{\theta_{1}}\left[g\left(\mathbf{X}_{n}^{+}\right) \frac{f_{\theta_{2}}^{+}\left(\mathbf{X}_{n}^{+}\right)}{f_{\theta_{1}}^{+}\left(\mathbf{X}_{n}^{+}\right)}\right] \\
& >\mathrm{E}_{\theta_{1}}\left[g\left(\mathbf{X}_{n}^{+}\right)\right] \mathrm{E}_{\theta_{1}}\left[\frac{f_{\theta_{2}}^{+}\left(\mathbf{X}_{n}^{+}\right)}{f_{\theta_{1}}^{+}\left(\mathbf{X}_{n}^{+}\right)}\right] \\
& =\mathrm{E}_{\theta_{1}}\left[g\left(\mathbf{X}_{n}^{+}\right)\right] .
\end{aligned}
$$

To apply the FKG inequality (51) with strict inequality it must be shown that (a) $f_{\theta_{1}}^{+}\left(\mathbf{x}_{n}\right)$ is FKG on $\mathbb{Z}_{+}^{n}$; and (b) the ratio $r\left(\mathbf{x}_{n}\right) \equiv \frac{f_{\theta_{2}}^{+}\left(\mathbf{x}_{n}\right)}{f_{\theta_{1}}^{+}\left(\mathbf{x}_{n}\right)}$ is strictly increasing on $\mathbb{Z}_{+}^{n} \cap\left\{f_{\theta_{1}}^{+}\left(\mathbf{x}_{n}\right)\right\}=\mathbb{Z}_{+}^{n} \cap\left\{h_{\mathbf{a}}\left(\mathbf{x}_{n}\right)>0\right\}$. First, for all $\theta>0$ and $\mathbf{x}_{n}>0$, it follows from (40) and (52) that

$$
f_{\theta}^{+}\left(\mathbf{x}_{n}\right)=\frac{b_{\theta, n} \theta^{x_{1}+\cdots+x_{n}}}{(A(\theta))^{x_{0}+\cdots+x_{n-1}}} \prod_{i=1}^{n} h_{\mathbf{a}}\left(x_{i-1}, x_{i}\right), \quad \mathbf{x}_{n}>0 .
$$

By TP2a each factor $h_{\mathbf{a}}\left(x_{i-1}, x_{i}\right)$ in (53) is TP2, hence their product is FKG, thus so is $f_{\theta}^{+}\left(\mathbf{x}_{n}\right)$; this gives (a). Next, $0<\theta_{1}<\theta_{2} \leq \tau \Rightarrow B\left(\theta_{1}\right)>B\left(\theta_{2}\right)$, so

$$
r\left(\mathbf{x}_{n}\right) \equiv \frac{b_{\theta_{2}, n}}{b_{\theta_{1}, n}}\left(\frac{A\left(\theta_{1}\right)}{A\left(\theta_{2}\right)}\right)^{x_{0}}\left(\frac{B\left(\theta_{1}\right)}{B\left(\theta_{2}\right)}\right)^{x_{1}+\cdots+x_{n-1}}\left(\frac{\theta_{2}}{\theta_{1}}\right)^{x_{n}}
$$

is strictly increasing in $x_{1}, \ldots, x_{n-1}, x_{n}$, which establishes (b).

(ii) The first inequality is immediate. For the second, apply the FKG 
inequality as follows:

$$
\begin{aligned}
\mathrm{E}_{\tau}\left[g\left(\ddot{\mathbf{X}}_{n}\right)\right] & =\mathrm{E}_{\tau}\left[g\left(\dot{\mathbf{X}}_{n}^{+}\right) \frac{\ddot{f}_{\tau}\left(\dot{\mathbf{X}}_{n}^{+}\right)}{\dot{f}_{\tau}^{+}\left(\dot{\mathbf{X}}_{n}^{+}\right)}\right] \\
& \geq \mathrm{E}_{\tau}\left[g\left(\dot{\mathbf{X}}_{n}^{+}\right)\right] \mathrm{E}_{\tau}\left[\frac{\ddot{f}_{\tau}\left(\dot{\mathbf{X}}_{n}^{+}\right)}{\dot{f}_{\tau}^{+}\left(\dot{\mathbf{X}}_{n}^{+}\right)}\right] \\
& =\mathrm{E}_{\tau}\left[g\left(\dot{\mathbf{X}}_{n}^{+}\right)\right] .
\end{aligned}
$$

As in (i), FKG is applicable in (55) because (a) $\dot{f}_{\tau}^{+}\left(\mathbf{x}_{n}\right) \equiv f_{\tau}^{+}\left(\mathbf{x}_{n}\right)$ is FKG on $\mathbb{Z}_{+}^{n}$ (by $(53)$ with $\theta=\tau$ ); and (b) the ratio

$$
r\left(\mathbf{x}_{n}\right) \equiv \frac{\ddot{f}_{\tau}\left(\mathbf{x}_{n}\right)}{\dot{f}_{\tau}^{+}\left(\mathbf{x}_{n}\right)}=\frac{x_{n}}{b_{\tau, n} x_{0}},
$$

(obtained from (47) and (52) with $\theta=\tau$ ) is increasing on $\mathbb{Z}_{+}^{n} \cap\left\{h_{\mathbf{a}}\left(\mathbf{x}_{n}\right)>0\right\}$.

To show that $\mathrm{E}_{\tau}\left[g\left(\ddot{\mathbf{X}}_{n}\right)\right]>\mathrm{E}_{\tau}\left[g\left(\dot{\mathbf{X}}_{n}^{+}\right)\right]$for at least one increasing $g$, take $g\left(\mathbf{x}_{n}\right)=1_{\{2,3, \ldots\}}\left(x_{1}\right)$. Because $\ddot{X}_{\tau, 1} \geq 1$ and $\dot{X}_{\tau, 1}^{+} \geq 1$, it follows from $(47)$ and (52) that

$$
\begin{aligned}
1-\mathrm{E}_{\tau}\left[g\left(\ddot{\mathbf{X}}_{n}\right)\right] & =\operatorname{Pr}_{\tau}\left[\ddot{X}_{1}=1\right] \\
& =\frac{\tau}{x_{0}(A(\tau))^{x_{0}}} h_{\mathbf{a}}\left(x_{0}, 1\right) \\
1-\mathrm{E}_{\tau}\left[g\left(\dot{\mathbf{X}}_{n}^{+}\right)\right]= & \operatorname{Pr}_{\tau}\left[\dot{X}_{1}^{+}=1\right] \\
= & \frac{\dot{b}_{1, \tau} \tau}{(A(\tau))^{x_{0}}} h_{\mathbf{a}}\left(x_{0}, 1\right) \\
= & \frac{\tau}{\left\{1-\operatorname{Pr}_{\tau}\left[\dot{X}_{1}=0\right]\right\}(A(\tau))^{x_{0}}} h_{\mathbf{a}}\left(x_{0}, 1\right) \\
= & \frac{\tau}{\left\{1-\left(\frac{a_{0}}{A(\tau)}\right)^{x_{0}}\right\}(A(\tau))^{x_{0}}} h_{\mathbf{a}}\left(x_{0}, 1\right) .
\end{aligned}
$$

Because $a_{0}>0$ and $x_{0} \geq 1$, we conclude that $\mathrm{E}_{\tau}\left[g\left(\ddot{\mathbf{X}}_{n}\right)\right]>\mathrm{E}_{\tau}\left[g\left(\dot{\mathbf{X}}_{n}^{+}\right)\right]$.

(iii) Since $B\left(\theta_{1}\right)<B\left(\theta_{2}\right)$ when $\tau \leq \theta_{1}<\theta_{2}$, the FKG inequality is not applicable here (recall (54)). Instead we use induction on $n$ to show that

$$
\mathrm{E}_{\theta}\left[g\left(\ddot{\mathbf{X}}_{n}\right)\right] \equiv \sum_{\mathbf{x}_{n}>0} g\left(\mathbf{x}_{n}\right) \ddot{f}_{\theta}\left(\mathbf{x}_{n}\right)
$$


is increasing for $\theta \in[\tau, \psi)$.

For $n=1$, (42) gives

$$
\ddot{f}_{\theta}\left(x_{1}\right)=\left(\frac{1-u_{\theta}^{x_{1}}}{1-u_{\theta}^{x_{0}}}\right) \frac{\theta^{x_{1}}}{(A(\theta))^{x_{0}}} h_{\mathbf{a}}\left(x_{0}, x_{1}\right), \quad x_{1}>0,
$$

which is TP2 in $\left(\theta, x_{1}\right)$ by TP2b, so

$$
\mathrm{E}_{\theta}\left[g\left(\ddot{X}_{1}\right)\right] \equiv \sum_{x_{1}>0} g\left(x_{1}\right) f_{\theta}\left(x_{1}\right)
$$

is increasing for $\theta \in(\tau, \psi)$ by the monotonicity-preserving property of a TP2 $\equiv$ MLR kernel (Karlin (1968, Ch.1, Proposition 3.1)). For $n \geq 2$,

$$
\begin{aligned}
\mathrm{E}_{\theta}\left[g\left(\ddot{\mathbf{X}}_{n}\right)\right] & =\mathrm{E}_{\theta}\left[\mathrm{E}_{\theta}\left[g\left(\ddot{\mathbf{X}}_{n-1}, \ddot{X}_{n}\right) \mid \ddot{\mathbf{X}}_{n-1}\right]\right] \\
& =\mathrm{E}_{\theta}\left[\sum_{x_{n}>0} g\left(\ddot{\mathbf{X}}_{n-1}, x_{n}\right) \ddot{f}_{\theta}\left(x_{n} \mid \ddot{X}_{n-1}\right)\right] \\
& \equiv \mathrm{E}_{\theta}\left[g_{\theta}^{*}\left(\ddot{\mathbf{X}}_{n-1}\right)\right] .
\end{aligned}
$$

From TP2a, TP2b, and (45), the transition probability $\ddot{f}_{\theta}\left(x_{n} \mid x_{n-1}\right)$ of the Markov process $\ddot{\mathbf{X}}_{\theta}$ is TP2 in $\left(x_{n}, \theta\right)$ and in $\left(x_{n}, x_{n-1}\right)$, so the monotonicitypreserving property implies that $g_{\theta}^{*}\left(\ddot{\mathbf{X}}_{n-1}\right)$ is increasing in $\theta$ and in $\ddot{\mathbf{X}}_{n-1}$. Thus by (60)-(61) and the induction hypothesis, $\mathrm{E}_{\theta}\left[g\left(\ddot{\mathbf{X}}_{n}\right)\right]$ is increasing for $\theta$ for $\theta \in(\tau, \psi)$. Lastly, these results extend to $[\tau, \psi)$ by $(49)$ and continuity.

To show that $\mathrm{E}_{\theta}\left[g\left(\ddot{\mathbf{X}}_{n}\right)\right]$ is strictly increasing in $\theta$ for at least one increasing $g$, take $g\left(\mathbf{x}_{n}\right)=1_{\{2,3, \ldots\}}\left(x_{1}\right)$. Because $\ddot{X}_{1} \geq 1$, it follows from (42) that

$$
\begin{aligned}
1-\mathrm{E}_{\theta}\left[g\left(\ddot{\mathbf{X}}_{n}\right)\right] & =\operatorname{Pr}_{\theta}\left[\ddot{X}_{1}=1\right] \\
& =\left(\frac{1-u_{\theta}}{1-u_{\theta}^{x_{0}}}\right) \frac{\theta}{(A(\theta))^{x_{0}}} h_{\mathbf{a}}\left(x_{0}, 1\right) \\
& =\left[\frac{\left(1-u_{\theta}\right) \theta}{\left(1-u_{\theta}^{x_{0}}\right) \theta^{x_{0}}}\right]\left[\frac{1}{B(\theta)}\right]^{x_{0}} h_{\mathbf{a}}\left(x_{0}, 1\right) .
\end{aligned}
$$

Because $x_{0} \geq 1$ the first factor in (62) is decreasing in $\theta$ by TP2b, while the second factor is strictly decreasing because $B(\theta)$ is strictly increasing for $\theta \in[\tau, \psi)$.

Lemma 4.3. Let $\mathbf{X}_{\theta}$ be a $G W$ branching process with psod offspring distribution (16). Each of the following two conditions is equivalent to Condition TP2b: for $\theta \in(\tau, \psi)$,

$$
\begin{aligned}
\frac{\mu_{\theta}-1}{1-\mu_{\theta u_{\theta}}} & \leq \frac{1}{u_{\theta}} \\
B^{\prime}\left(\theta u_{\theta}\right)+B^{\prime}(\theta) & \leq 0
\end{aligned}
$$


Proof. Let $\delta_{\theta}=d u_{\theta} / d \theta$. Then $\left(1-u_{\theta}^{x}\right) \theta^{x}$ is TP2 iff for $x=1,2, \ldots$, the ratio $\frac{\left(1-u_{\theta}^{x+1}\right) \theta^{x+1}}{\left(1-u_{\theta}^{x}\right) \theta^{x}}$ is increasing in $\theta$ for $\theta \in(\tau, \psi)$, equivalently, iff

$$
\frac{d}{d \theta} \log \left[\frac{\left(1-u_{\theta}^{x+1}\right) \theta}{\left(1-u_{\theta}^{x}\right)}\right] \equiv \frac{-(x+1) u_{\theta}^{x} \delta_{\theta}}{1-u_{\theta}^{x+1}}+\frac{x u_{\theta}^{x-1} \delta_{\theta}}{1-u_{\theta}^{x}}+\frac{1}{\theta} \geq 0 .
$$

After some algebra, we find that this is equivalent to the inequality

$$
\left[\left(1-u^{x}\right)-x u^{x}(1-u)\right]+d_{\theta} u^{x-1}\left[x(1-u)-u\left(1-u^{x}\right)\right] \geq 0,
$$

where we use the relation $d_{\theta}=\theta \delta_{\theta}+u_{\theta}$ and abbreviate $u_{\theta}$ by $u$. Because both terms in square brackets are positive and $d_{\theta}<0$, this is in turn equivalent to the inequality

$$
-d_{\theta} \leq \frac{\left(1-u^{x}\right)-x u^{x}(1-u)}{u^{x-1}\left[x(1-u)-u\left(1-u^{x}\right)\right]} \equiv \Delta(u, x) .
$$

But $\Delta(u, 1)=1$ and $\Delta(u, x) \geq 1$ for $x \geq 2$ :

$$
\begin{aligned}
\Delta(u, x)-1 & =\frac{\left(1-u^{x}\right)\left(1+u^{x}\right)-u^{x-1} x(1-u)(1+u)}{u^{x-1}\left[x(1-u)-u\left(1-u^{x}\right)\right]} \\
& =\frac{\left(1-u^{2 x}\right)-u^{x-1} x\left(1-u^{2}\right)}{u^{x-1}\left[x(1-u)-u\left(1-u^{x}\right)\right]} \\
& =\frac{\left(1-u^{2}\right)\left[\left(1+u^{2}+\cdots+u^{2(x-1)}\right)-u^{2(x-1) / 2} x\right]}{u^{x-1}\left[x(1-u)-u\left(1-u^{x}\right)\right]} \\
& \geq 0
\end{aligned}
$$

because $u^{2 x}$ is convex in $x$. Thus TP2b is equivalent to the simple relation

$$
-d_{\theta} \leq \Delta(u, 1) \equiv 1
$$

which, by (27), is equivalent to (63). Lastly, differentiate (23) with respect to $\theta$ to establish the equivalence of (68) and (64).

Example 4.1 (= 3.1 continued). For the Poisson $(\theta)$ psod, the coefficient of $\theta^{y}$ in the power series $[A(\theta)]^{x}=e^{x \theta}$ is $h_{\mathbf{a}}(x, y)=x^{y} / y$ !, which is TP2 in $(x, y)$ so TP2a is satisfied. Furthermore $\mu_{\theta}=\theta, \tau=1$, and from (33),

$$
-\frac{\log u_{\theta}}{1-u_{\theta}}=\theta
$$


for $\theta \geq 1$, so (63) is equivalent to the inequality

$$
-2 u \log u \leq 1-u^{2}
$$

where $u=u_{\theta}$. This inequality holds for all $u \in[0,1]$, hence TP2b is also satisfied. Thus by Proposition 4.2, $\left(\dot{\mathbf{X}}_{\theta}\right)_{n}^{+}$is stochastically decreasing and $\left(\ddot{\mathbf{X}}_{\theta}\right)_{n}$ is stochastically increasing for $\theta \geq 1$, while $\left(\dot{\mathbf{X}}_{\tau}\right)_{n}^{+} \prec\left(\ddot{\mathbf{X}}_{\tau}\right)_{n}$.

Example 4.2 (= 3.2 continued). For the negative $\operatorname{binomial}(r, \theta) \operatorname{psod}$, the coefficient of $\theta^{y}$ in the power series $[A(\theta)]^{x}=1 /(1-\theta)^{r x}$ is

$$
h_{\mathbf{a}}(x, y)=\frac{\Gamma(r x+y)}{\Gamma(r x) y !}=\frac{(r x+y-1) \cdots(r x)}{y !},
$$

which is TP2 in $(x, y)$, so $\mathrm{NB}(r, \cdot)$ satisfies TP2a for all $r>0$. Next, $\mu_{\theta}=$ $\frac{r \theta}{(1-\theta)}$ and $\tau=\frac{t}{1+t}$, where $t=\frac{1}{r}$. Set $u=u_{\theta}$ and apply (35) to obtain

$$
\begin{aligned}
\frac{1-\theta}{1-\theta u} & =u^{t}, \\
\frac{1-u^{t}}{1-u^{t+1}} & =\theta .
\end{aligned}
$$

After some algebra it is seen that (63) is equivalent to each of the inequalities

$$
\begin{aligned}
& \frac{1-u^{t}}{1-u^{t+1}} \leq \tau\left(\frac{1+u^{t-1}}{1+u^{t}}\right), \\
& \frac{v^{t}-v^{-t}}{t} \leq v-v^{-1}
\end{aligned}
$$

where $v=u^{-1} \geq 1$. Because $h(t) \equiv v^{t}-v^{-t}$ is convex in $t$ and $h(0)=0,(75)$ holds iff $t \leq 1$. Thus the $\mathrm{NB}(r, \cdot)$ psod family satisfies TP2b iff $r \geq 1$. This includes the geometric psod family $(r=t=1)$ where equality holds in (75).

Thus by Proposition 4.2, if $r \geq 1$ then $\left(\dot{\mathbf{X}}_{\theta}\right)_{n}^{+}$is stochastically decreasing and $\left(\ddot{\mathbf{X}}_{\theta}\right)_{n}$ is stochastically increasing for $\tau \leq \theta<1$, while $\left(\dot{\mathbf{X}}_{\tau}\right)_{n}^{+} \prec\left(\ddot{\mathbf{X}}_{\tau}\right)_{n}$, where $\tau=1 /(1+r)$.

Example 4.3 (= 3.3 continued). For the binary splitting GW process, the coefficient of $\theta^{y}$ in the power series $[A(\theta)]^{x}=\left(1+\theta^{2}\right)^{x}$ is

$$
h_{\mathbf{a}}(x, y)=\left\{\begin{array}{cl}
\left(\begin{array}{l}
x \\
\frac{y}{2}
\end{array}\right) & \text { for } y=0,2, \ldots, 2 x, \\
0 & \text { otherwise }
\end{array}\right.
$$


which is TP2 in $(x, y)$, so TP2a is satisfied. Furthermore $B(\theta)=\theta+\theta^{-1}$ and $u_{\theta}=\theta^{-2}$ for $\theta \geq \tau=1$, so (64) is equivalent to the valid inequality $2-\theta^{2}-\theta^{-2} \leq 0$, hence TP2b is satisfied. Thus by Proposition $4.2,\left(\dot{\mathbf{X}}_{\theta}\right)_{n}^{+}$ is stochastically decreasing and $\left(\ddot{\mathbf{X}}_{\theta}\right)_{n}$ is stochastically increasing for $\theta \geq 1$, while $\left(\dot{\mathbf{X}}_{\tau}\right)_{n}^{+} \prec\left(\ddot{\mathbf{X}}_{\tau}\right)_{n}$.

Remark 4.1. The maximum likelihood estimate (MLE) $\hat{\theta}$ is derived by differentiating (40), then applying (18) to obtain the relation

$$
\hat{\mu} \equiv \mu_{\hat{\theta}}=\frac{Y_{n}-x_{0}}{Y_{n-1}},
$$

from which $\hat{\theta}$ can be obtained. Here $\hat{\mu}$ denotes the MLE of the mean $\mu_{\theta}$.

\section{Predicting extinction or explosion: the fixed sample size case}

Based on observed data $\mathbf{x}_{n} \equiv\left(x_{1}, \ldots, x_{n}\right)$ from a non-terminated psod $G W$ process $\mathbf{X} \equiv \mathbf{X}_{\theta}$ with initial size $x_{0}$ and fixed $n$, predict whether extinction or explosion will occur for the current realization of the process.

By the Markov property for $\mathbf{X}$,

$$
\operatorname{Pr}_{\theta}\left[\text { extinction } \mid \mathbf{X}_{n}=\mathbf{x}_{n}\right]=u_{\theta}^{x_{n}}=1-\operatorname{Pr}_{\theta}\left[\text { explosion } \mid \mathbf{X}_{n}=\mathbf{x}_{n}\right]
$$

The MLE $\hat{u}$ of $u_{\theta}$ is given by $\hat{u}=u_{\hat{\theta}}$ where $\hat{\theta}$ is obtained from (77), so the estimated extinction probability is

$$
\operatorname{Pr}_{\hat{\theta}}\left[\text { extinction } \mid \mathbf{X}_{n}=\mathbf{x}_{n}\right]=\hat{u}^{x_{n}} \begin{cases}=1 & \text { if } x_{n} \leq x_{0} \\ <1 & \text { if } x_{n}>x_{0}\end{cases}
$$

The value of $\hat{u}^{x_{n}}$ can be used to predict extinction or explosion.

However, this procedure may reach unwarranted conclusions. For example, if $x_{n}=x_{0}-1$ then extinction will be predicted with certainty even though the population has declined only slightly. Whereas (79) is based solely on the value of $x_{n}$, the prediction procedures derived in this section compare $x_{n}$ to $n$ in order to predict whether the observed process is on a trajectory toward extinction or toward explosion.

5.1. Prediction as a testing problem. We reformulate the prediction problem as a hypothesis-testing problem to which Neyman-Pearson theory can be applied. As in Proposition 4.2, let $\mathbf{X}_{n}^{+} \equiv\left(\mathbf{X}_{\theta}\right)_{n}^{+}$denote the conditional 
random vector $\mathbf{X}_{n} \mid X_{n}>0$. Then conditional on non-termination at time $n$, extinction will occur iff either

$$
H_{\leq}^{+}: \mathbf{X}_{n}^{+} \stackrel{d}{=}\left(\mathbf{X}_{\theta}\right)_{n}^{+}, \theta \leq \tau \quad \text { or } \quad \dot{H}_{\geq}^{+}: \mathbf{X}_{n}^{+} \stackrel{d}{=}\left(\dot{\mathbf{X}}_{\theta}\right)_{n}^{+}, \theta \geq \tau
$$

holds, while explosion will occur iff $\ddot{H}_{>}^{+}: \mathbf{X}_{n}^{+} \stackrel{d}{=}\left(\ddot{\mathbf{X}}_{\theta}\right)_{n}^{+}, \theta>\tau$ holds. (Recall that $\left(\ddot{\mathbf{X}}_{\theta}\right)^{+}=\ddot{\mathbf{X}}_{\theta}$.) However, $H_{\leq}^{+}=\dot{H}_{\geq}^{+}$by Proposition 3.2 while by (49) the $L_{1}$-closure of $\ddot{H}_{>}^{+}$is

$$
\ddot{H}_{\geq}^{+}: \mathbf{X}_{n}^{+} \stackrel{d}{=}\left(\ddot{\mathbf{X}}_{\theta}\right)_{n}^{+}, \theta \geq \tau
$$

so the prediction problem can be formulated as the following testing problem:

Based on observing $\mathbf{X}_{n}^{+}=\mathbf{x}_{n}>0$, test

$$
\left.\dot{H}_{\geq}^{+}(\text {eventual extinction }) \quad \text { vs. } \ddot{H}_{\geq}^{+} \text {(eventual explosion }\right) .
$$

Either $\dot{H}_{\geq}^{+}$or $\ddot{H}_{\geq}^{+}$may be taken to be the null hypothesis. Note that $\theta \geq \tau$ under both $\dot{H}_{\geq}^{+}$and $\ddot{H}_{\geq}^{+}$. The conditional pmfs of $\mathbf{X}_{n}^{+}$under $\dot{H}_{\geq}^{+}$and $\ddot{H}_{\geq}^{+}$are

$$
\dot{f}_{\theta}^{+}\left(\mathbf{x}_{n}\right) \equiv \operatorname{Pr}_{\theta}\left[\dot{\mathbf{X}}_{n}^{+}=\mathbf{x}_{n}\right]=\dot{b}_{\theta, n} \dot{f}_{\theta}\left(\mathbf{x}_{n}\right), \quad \mathbf{x}_{n}>0
$$

and $\ddot{f}_{\theta}^{+}\left(\mathbf{x}_{n}\right) \equiv \ddot{f}_{\theta}\left(\mathbf{x}_{n}\right)$, respectively, where $\ddot{f}_{\theta}$ is given by (42) and (47) and

$$
\dot{b}_{\theta, n}^{-1}=\operatorname{Pr}_{\theta}\left[\dot{X}_{n}>0\right]=\operatorname{Pr}_{\theta u_{\theta}}\left[X_{n}>0\right]=b_{\theta u_{\theta}, n}^{-1} .
$$

A version of the generalized LR criterion for (81) is

$$
\lambda^{+}\left(\mathbf{x}_{n}\right) \equiv \frac{\sup _{\tau \leq \theta<\psi} \ddot{f}_{\theta}^{+}\left(\mathbf{x}_{n}\right)}{\sup _{\tau \leq \theta<\psi} \dot{f}_{\theta}^{+}\left(\mathbf{x}_{n}\right)}
$$

but the numerator and denominator may be difficult to evaluate.

5.2. The least favorable distributions for fixed sample size. When the psod satisfies Conditions TP2a and TP2b the testing problem (81) has a convenient solution. Proposition 4.2 implies that $\dot{H}_{\geq}^{+}$and $\ddot{H}_{\geq}$are separated families and that $\left(\dot{f}_{\tau}^{+}, \ddot{f}_{\tau}^{+}\right)$is a pair of least favorable distributions for (81). By Theorem 3.8.1 of Lehmann and Romano (2005), a test of the form

$$
\begin{cases}\text { accept } \left.\dot{H}_{\geq}^{+} \text {(predict extinction }\right) & \text { if } \lambda_{\tau}^{+}\left(\mathbf{X}_{n}^{+}\right) \leq d \\ \text { accept } \left.\ddot{H}_{\geq} \text {(predict explosion }\right) & \text { if } \lambda_{\tau}^{+}\left(\mathbf{X}_{n}^{+}\right)>d\end{cases}
$$


is the UMP test of its size for (81), where $d$ is a nonnegative constant and, from (47) and (82),

$$
\lambda_{\tau}^{+}\left(\mathbf{x}_{n}\right) \equiv \frac{\ddot{f}_{\tau}^{+}\left(\mathbf{x}_{n}\right)}{\dot{f}_{\tau}^{+}\left(\mathbf{x}_{n}\right)}=\frac{x_{n}}{x_{0} \dot{b}_{\tau, n}}=\frac{x_{n}}{x_{0} b_{\tau, n}}, \quad \mathbf{x}_{n}>0 .
$$

Because $\lambda_{\tau}^{+}\left(\mathbf{x}_{n}\right)$ is strictly increasing in $x_{n}$, the test (85) has the form

$$
\begin{cases}\text { accept } \left.\dot{H}_{\geq}^{+} \text {(predict extinction }\right) & \text { if } X_{n}^{+} \leq c, \\ \text { accept } \ddot{H}_{\geq}^{+} \text {(predict explosion) } & \text { if } X_{n}^{+} \geq c+1\end{cases}
$$

where $c$ is a nonnegative integer.

5.3. Exponential-type approximations for $\dot{X}_{n}^{+}$when $\theta=\tau$. Suppose first that $\dot{H}_{\geq}^{+}$is taken to be the null hypothesis. If $X_{n}^{+}=x_{n}>0$ is observed, the $p$-value $\operatorname{Pr}_{\tau}\left[\dot{X}_{n}^{+} \geq x_{n}\right]$ for test (87) is determined by the distribution of $\dot{X}_{n}^{+}$under $\dot{f}_{\tau}^{+}$. For large $n$ the mean and variance of $\dot{X}_{n}^{+}$can be approximated via (93) as follows:

$$
\begin{aligned}
\mathrm{E}_{\tau}\left(\dot{X}_{n}^{+}\right) & =x_{0} b_{\tau, n} \approx \frac{n \sigma_{\tau}^{2}}{2} \\
\operatorname{Var}_{\tau}\left(\dot{X}_{n}^{+}\right) & =x_{0} b_{\tau, n}\left[n \sigma_{\tau}^{2}-x_{0}\left(b_{\tau, n}-1\right)\right] \approx \frac{n \sigma_{\tau}^{2}}{2}\left(\frac{n \sigma_{\tau}^{2}}{2}+x_{0}\right) .
\end{aligned}
$$

Unfortunately the conditional rv $\dot{X}_{n}^{+}\left(\equiv \dot{X}_{n} \mid X_{n}>0\right)$ is not the sum of $x_{0}$ i.i.d. copies each with initial size 1: conditional on $X_{n}>0$, some of the initial $x_{0}$ family lines may have terminated by time $n$. Therefore a normal approximation is not available for $\dot{X}_{n}^{+}$, even when $x_{0}$ is large.

Fortunately, however, when $n$ is large Yaglom's classical exponential approximation can be applied. For a critical GW process (not necessarily psod) with offspring variance $\sigma^{2}<\infty$, Yaglom (1947) showed that if $x_{0}=1$ then

$$
\lim _{n \rightarrow \infty} \operatorname{Pr}\left[X_{n}^{+} \geq n z\right]=e^{-2 z / \sigma^{2}}
$$

for any $z>0$. This result appears under progressively weaker moment conditions in Harris (1963, §I.10), Kesten, Ney, and Spitzer (1966, p.582), Athreya and Ney (1972, §9), and Jagers (1975, Theorem 2.4.2), but only for the case $x_{0}=1$. When $x_{0} \geq 2$ it might be expected that the limiting exponential (EXP) distribution in (90) should be replaced by the distribution 
of the sum of $x_{0}$ independent exponential rvs, i.e., a gamma distribution, but (90) continues to hold without change, cf. (92). However, we will also present a more accurate gamma (GAM) approximation (94) that does depend on $x_{0}$.

Let $G_{r}$ denote a gamma rv with shape parameter $r>0$ and scale parameter 1 and let $G_{r}(z)$ denote its cdf, that is,

$$
G_{r}(z)=\frac{1}{\Gamma(r)} \int_{0}^{z} t^{r-1} e^{-t} d t
$$

Proposition 5.1. (i) Let $\left\{X_{n}\right\}$ be a critical GW process with offspring pgf $\phi$, offspring variance $\sigma^{2}<\infty$, and initial size $x_{0} \geq 1$. For any $z>0$,

$$
\begin{aligned}
& \lim _{n \rightarrow \infty} \operatorname{Pr}\left[X_{n}^{+} \geq n z\right]=e^{-2 z / \sigma^{2}}, \\
& \lim _{n \rightarrow \infty} n \operatorname{Pr}\left[X_{n}>0\right]=2 x_{0} / \sigma^{2} .
\end{aligned}
$$

(ii) Let $\bar{G}_{r}(z)=1-G_{r}(z)$. For $x_{0} \geq 1$ and large $n$,

$$
\operatorname{Pr}\left[X_{n}^{+} \geq n z\right] \approx \frac{1}{1-\left(1-\frac{2}{n \sigma^{2}}\right)^{x_{0}}} \sum_{r=1}^{x_{0}}\left(\begin{array}{c}
x_{0} \\
r
\end{array}\right) \bar{G}_{r}\left(\frac{2 z}{\sigma^{2}}\right)\left(\frac{2}{n \sigma^{2}}\right)^{r}\left(1-\frac{2}{n \sigma^{2}}\right)^{x_{0}-r} .
$$

Proof. (i) The existing results for the case $x_{0}=1$ are based on the following fact, cf. Jagers (1975, Lemma 2.4.1):

$$
\lim _{n \rightarrow \infty} \frac{1}{n}\left[\frac{1}{1-\phi_{n}(s)}-\frac{1}{1-s}\right]=\frac{\sigma^{2}}{2} \quad \text { uniformly for } 0 \leq s<1 .
$$

Set $s=0$ to obtain

$$
\lim _{n \rightarrow \infty} n\left(1-\phi_{n}(0)\right)=\frac{2}{\sigma^{2}} .
$$

For $x_{0} \geq 2, X_{n}$ has pgf $\phi_{n}^{x_{0}}$ so by $(96)$,

$$
\begin{aligned}
n \operatorname{Pr}\left[X_{n}>0\right] & =n\left(1-\phi_{n}^{x_{0}}(0)\right) \\
& =n\left(1-\phi_{n}(0)\right)\left[1+\phi_{n}(0)+\cdots+\phi_{n}^{x_{0}-1}(0)\right] \\
& \rightarrow \frac{2 x_{0}}{\sigma^{2}}
\end{aligned}
$$

because $\phi_{n}(0) \uparrow 1$; this confirms (93). Furthermore, the Laplace transform 
of $X_{n}^{+} / n$ is, for $t \geq 0$

$$
\begin{aligned}
& \mathrm{E}\left[e^{-t X_{n}^{+} / n}\right] \\
= & \frac{\phi_{n}^{x_{0}}\left(e^{-t / n}\right)-\phi_{n}^{x_{0}}(0)}{1-\phi_{n}^{x_{0}}(0)} \\
= & 1-\frac{1-\phi_{n}^{x_{0}}\left(e^{-t / n}\right)}{1-\phi_{n}^{x_{0}}(0)} \\
= & 1-\frac{n\left(1-\phi_{n}\left(e^{-t / n}\right)\right)\left[1+\phi_{n}\left(e^{-t / n}\right)+\cdots+\phi_{n}^{x_{0}-1}\left(e^{-t / n}\right)\right]}{n\left(1-\phi_{n}(0)\right)\left[1+\phi_{n}(0)+\cdots+\phi_{n}^{x_{0}-1}(0)\right]} \\
\rightarrow & 1-\frac{\frac{\sigma^{2}}{2}}{\frac{1}{t}+\frac{\sigma^{2}}{2}} \frac{x_{0}}{x_{0}}=\frac{1}{1+\frac{t \sigma^{2}}{2}} \quad \text { as } n \rightarrow \infty
\end{aligned}
$$

by $(95)$ and the inequalities $\phi_{n}(0)<\phi_{n}\left(e^{-t / n}\right)<1$. This is the Laplace transform of the exponential distribution in (92), confirming that result.

(ii) Represent $X_{n} \stackrel{d}{=} U_{1}+\cdots+U_{x_{0}}$, where the $U_{i}$ are i.i.d. copies of $X_{n}$ but each with initial size $x_{0}=1$. Then

$$
\begin{aligned}
& \operatorname{Pr}\left[X_{n}>0\right] \operatorname{Pr}\left[X_{n}^{+} \geq n z\right] \\
& \equiv \operatorname{Pr}\left[U_{1}+\cdots U_{x_{0}}>0\right] \operatorname{Pr}\left[\left(U_{1}+\cdots+U_{x_{0}}\right)^{+} \geq n z\right] \\
& =\operatorname{Pr}\left[U_{1}+\cdots+U_{x_{0}} \geq n z\right] \\
& =\sum_{\omega \in 2^{x_{0}} \backslash \emptyset} \operatorname{Pr}\left[\sum_{i \in \omega} U_{i} \geq n z, U_{i}>0 \text { for } i \in \omega, U_{i}=0 \text { for } i \notin \omega\right] \\
& =\sum_{r=1}^{x_{0}}\left(\begin{array}{c}
x_{0} \\
r
\end{array}\right) \operatorname{Pr}\left[U_{1}+\cdots+U_{r} \geq n z, U_{1}>0, \ldots, U_{r}>0, U_{r+1}=\cdots=U_{x_{0}}=0\right] \\
& =\sum_{r=1}^{x_{0}}\left(\begin{array}{c}
x_{0} \\
r
\end{array}\right) \operatorname{Pr}\left[U_{1}^{+}+\cdots+U_{r}^{+} \geq n z\right] \operatorname{Pr}\left[U_{1}>0\right]^{r} \operatorname{Pr}\left[U_{x_{0}}=0\right]^{x_{0}-r} \\
& \approx \sum_{r=1}^{x_{0}}\left(\begin{array}{c}
x_{0} \\
r
\end{array}\right) \bar{G}_{r}\left(\frac{2 z}{\sigma^{2}}\right)\left(\frac{2}{n \sigma^{2}}\right)^{r}\left(1-\frac{2}{n \sigma^{2}}\right)^{x_{0}-r}
\end{aligned}
$$

for large $n$, by (92) and by (93) with $x_{0}=1$. Furthermore, by (97) and (96),

$$
\operatorname{Pr}\left[X_{n}>0\right]=\left(1-\phi_{n}^{x_{0}}(0)\right) \approx 1-\left(1-\frac{2}{n \sigma^{2}}\right)^{x_{0}},
$$

which yields (94). 
From (92) and (94) and a continuity correction we obtain exponentialtype approximations for the $p$-value of the test $(87)$ when $\dot{H}_{\geq}^{+}$and $\ddot{H}_{\geq}^{+}$are taken to be the null and alternative hypothesis, respectively:

$$
\begin{aligned}
\operatorname{Pr}_{\tau}\left[\dot{X}_{n}^{+} \geq x_{n}\right] & \approx e^{-\frac{2\left(x_{n}-1\right)}{n \sigma_{\tau}^{2}}} \\
& \equiv \dot{\pi}^{\operatorname{EXP}}\left(x_{n} ; n\right), \\
\operatorname{Pr}_{\tau}\left[\dot{X}_{n}^{+} \geq x_{n}\right] & \approx \frac{1}{1-\left(1-\frac{2}{n \sigma_{\tau}^{2}}\right)^{x_{0}}} \sum_{r=1}^{x_{0}}\left(\begin{array}{c}
x_{0} \\
r
\end{array}\right)\left(\frac{2}{n \sigma_{\tau}^{2}}\right)^{r}\left(1-\frac{2}{n \sigma_{\tau}^{2}}\right)^{x_{0}-r} \bar{G}_{r}\left(\frac{2\left(x_{n}-1\right)}{n \sigma_{\tau}^{2}}\right) \\
& \equiv \dot{\pi}^{\operatorname{GAM}}\left(x_{n} ; n, x_{0}\right) .
\end{aligned}
$$

The EXP and GAM approximations coincide when $x_{0}=1$. The approximate $p$-value $\dot{\pi}^{\mathrm{EXP}}\left(x_{n} ; n\right)$ does not depend on the value of $x_{0}$; it conveys significance for $\ddot{H}_{>}^{+}$(explosion) iff $x_{n} \gg n \sigma_{\tau}^{2}$, but convergence to the exact $p$-value is slow, see Remark 5.1. The $\dot{\pi}^{\mathrm{GAM}}\left(x_{n} ; n\right)$ approximation is noticeably better.

Remark 5.1. The accuracy of the EXP and GAM approximations can be assessed for the geometric psod (cf. Example 3.2). Here the pgf of $X_{n} \equiv \dot{X}_{n}$ in the critical case can be obtained explicitly ${ }^{8}$ and expanded in a power series, from which the exact distribution of $X_{n}$ can be recovered. By (82) and (83) this yields the exact distribution of $X_{n}^{+} \equiv \dot{X}_{n}^{+}$in the critical case. The exact $p$-values and their approximations $\dot{\pi}^{\mathrm{EXP}}$ and $\dot{\pi}^{\mathrm{GAM}}$ are shown in Tables 1 and 2 , from which the superiority of the GAM approximation is apparent.

5.4. Exponential-type approximations for $\ddot{X}_{n}^{+}$when $\theta=\tau$. Suppose next that $\ddot{H}_{\geq}^{+}$is the null hypothesis. The $p$-value $\operatorname{Pr}_{\tau}\left[\ddot{X}_{n}^{+} \leq x_{n}\right]$ for test (87) is determined by the distribution of $\ddot{X}_{n}^{+} \equiv \ddot{X}_{n}$ under $\ddot{f}_{\tau}^{+} \equiv \ddot{f}_{\tau}$. Again a normal approximation is not available for large $x_{0}$ because $\ddot{X}_{n}$ is not the sum of $x_{0}$ i.i.d. copies each with initial size 1: conditional on explosion, some of the initial $x_{0}$ family lines nonetheless may become extinct. However, an exponential-type approximation is available for large $n$, based on the following representation for the process $\ddot{\mathbf{X}}_{\tau} \equiv\left\{\ddot{X}_{\tau, n} \mid n \geq 1\right\}$. We shall abbreviate $\ddot{X}_{\tau, n}$ to $\ddot{X}_{n}$.

Proposition 5.2 Define $Z_{n}=\ddot{X}_{n}-1, n=1,2, \ldots, z_{0}=x_{0}-1$. When $\theta=\tau, \mathbf{Z} \equiv\left\{Z_{n} \mid n \geq 1\right\}$ is a critical $G W$ process with immigration (GWI). Specifically,

$$
Z_{n} \mid Z_{n-1}=\xi_{1}^{(n)}+\cdots+\xi_{Z_{n-1}}^{(n)}+W_{n}
$$

\footnotetext{
${ }^{8}$ cf. eqn.(8.32) in Taylor and Karlin (1998) for the case $x_{0}=1$.
} 


\begin{tabular}{|lll|c|cc|}
\hline & & & $x_{0}=1$ & \multicolumn{2}{|c|}{$x_{0}=2$} \\
\hline$n$ & $x_{n}$ & $\dot{\pi}^{\text {EXP }}$ & Exact & $\dot{\pi}^{\text {GAM }}$ & Exact \\
\hline 5 & 10 & 0.165 & 0.194 & 0.198 & 0.226 \\
5 & 20 & 0.022 & 0.031 & 0.032 & 0.042 \\
5 & 30 & 0.003 & 0.005 & 0.005 & 0.008 \\
\hline 10 & 20 & 0.150 & 0.164 & 0.165 & 0.178 \\
10 & 40 & 0.020 & 0.024 & 0.024 & 0.029 \\
10 & 60 & 0.003 & 0.004 & 0.004 & 0.005 \\
\hline 15 & 20 & 0.282 & 0.293 & 0.294 & 0.305 \\
15 & 40 & 0.074 & 0.081 & 0.081 & 0.087 \\
15 & 60 & 0.020 & 0.022 & 0.022 & 0.025 \\
\hline 100 & 150 & 0.225 & 0.227 & 0.227 & 0.229 \\
\hline
\end{tabular}

Table 1: Exact and approximate $p$-values $\operatorname{Pr}_{\tau}\left[\dot{X}_{n}^{+} \geq x_{n}\right]$ for the geometric psod when $x_{0}=1$ and $x_{0}=2$.

\begin{tabular}{|lll|ll|ll|}
\hline & & & \multicolumn{2}{|c|}{$x_{0}=8$} & \multicolumn{2}{c|}{$x_{0}=14$} \\
\hline$n$ & $x_{n}$ & $\dot{\pi}^{\mathrm{EXP}}$ & $\dot{\pi}^{\mathrm{GAM}}$ & Exact & $\dot{\pi}^{\mathrm{GAM}}$ & Exact \\
\hline 5 & 10 & 0.165 & 0.420 & 0.428 & 0.631 & 0.618 \\
5 & 20 & 0.022 & 0.129 & 0.141 & 0.286 & 0.288 \\
5 & 30 & 0.003 & 0.035 & 0.042 & 0.108 & 0.115 \\
\hline 10 & 20 & 0.150 & 0.262 & 0.273 & 0.369 & 0.375 \\
10 & 40 & 0.020 & 0.058 & 0.064 & 0.108 & 0.115 \\
10 & 60 & 0.003 & 0.012 & 0.014 & 0.029 & 0.032 \\
\hline 15 & 20 & 0.282 & 0.369 & 0.378 & 0.444 & 0.450 \\
15 & 40 & 0.074 & 0.126 & 0.133 & 0.178 & 0.184 \\
15 & 60 & 0.020 & 0.042 & 0.046 & 0.069 & 0.073 \\
\hline 100 & 150 & 0.225 & 0.237 & 0.239 & 0.248 & 0.249 \\
\hline
\end{tabular}

Table 2: Exact and approximate $p$-values $\operatorname{Pr}_{\tau}\left[\dot{X}_{n}^{+} \geq x_{n}\right]$ for the geometric psod when $x_{0}=8$ and $x_{0}=14$.

where $\xi_{1}^{(n)}, \ldots, \xi_{Z_{n-1}}^{(n)}, W_{n}$ are independent rvs, $\xi_{j}^{(n)} \stackrel{d}{=} \xi_{\tau}$, and $W_{n}$ is a nonnegative integer-valued $r v$ with $p g f \phi_{\tau}^{\prime}(s)$. (This is a pgf since $\phi_{\tau}^{\prime}(1)=\mu_{\tau}=1$.) 
Proof. From (47),

$$
\begin{aligned}
\ddot{f}_{\tau}\left(\mathbf{x}_{n}\right) & =\prod_{i=1}^{n} \frac{x_{i}}{x_{i-1}} \frac{\tau^{x_{i}}}{(A(\tau))^{x_{i-1}}} h_{\mathbf{a}}\left(x_{i-1}, x_{i}\right) \\
& \equiv \prod_{i=1}^{n} g_{\tau}\left(x_{i} \mid x_{i-1}\right),
\end{aligned}
$$

so $\ddot{\mathbf{X}}_{\tau}$ is a Markov chain with transition probability $g_{\tau}\left(x_{i} \mid x_{i-1}\right)$. The conditional pgf corresponding to $g_{\tau}\left(x_{i} \mid x_{i-1}\right)$ is

$$
\begin{aligned}
\mathrm{E}_{\tau}\left(s^{\ddot{X}_{i}} \mid \ddot{X}_{i-1}=\ddot{x}_{i-1}\right) & =\frac{1}{x_{i-1}} \sum_{x_{i}} x_{i} s^{x_{i}} \frac{\tau^{x_{i}}}{(A(\tau))^{x_{i-1}}} h_{\mathbf{a}}\left(x_{i-1}, x_{i}\right) \\
& =\frac{s}{x_{i-1}} \frac{d}{d s} \sum_{x_{i}} s^{x_{i}} \frac{\tau^{x_{i}}}{(A(\tau))^{x_{i-1}}} h_{\mathbf{a}}\left(x_{i-1}, x_{i}\right) \\
& =\frac{s}{x_{i-1}} \frac{d}{d s}\left[\left(\phi_{\tau}(s)\right)^{x_{i-1}}\right] \\
& =s\left(\phi_{\tau}(s)\right)^{x_{i-1}-1} \phi_{\tau}^{\prime}(s) .
\end{aligned}
$$

The third equality holds since $\frac{\tau^{x_{i}}}{(A(\tau))^{x_{i-1}}} h_{\mathbf{a}}\left(x_{i-1}, x_{i}\right)$ is the pmf of $\xi_{1}^{(i)}+\cdots+$ $\xi_{x_{i-1}}^{(i)}$. Thus (105) implies that

$$
\ddot{X}_{i} \mid \ddot{X}_{i-1}=1+\xi_{1}^{(i)}+\cdots+\xi_{X_{i-1}-1}^{(i)}+W_{i},
$$

where the $\xi_{j}^{(i)}$ 's and $W_{i}$ are mutually independent rvs, the $\xi_{j}^{(i)}$ 's have common $\operatorname{pgf} \phi_{\tau}$, and $W_{i}$ is the nonnegative integer-valued rv with $\operatorname{pgf} \phi_{\tau}^{\prime}(s)$. Now set $i=n$ in (106) to obtain (103).

By the theorem of Seneta (1970), $2 Z_{n} / n \sigma_{\tau}^{2} \stackrel{d}{\rightarrow} G_{2}$ (cf. (91)) if $z_{0}=1$ $\left(x_{0}=2\right)$. Since $\ddot{X}_{n}=Z_{n}+1$, we obtain the following approximation when $x_{0}=2$ :

$$
\operatorname{Pr}_{\tau}\left[\ddot{X}_{n} \leq x_{n}\right] \approx G_{2}\left(\frac{2 x_{n}}{n \sigma_{\tau}^{2}}\right) \equiv \ddot{\pi}^{\mathrm{G} 2}\left(x_{n} ; n\right) \text { for large } n .
$$

We now show that if $n$ is sufficiently large, $\ddot{\pi}^{\mathrm{G} 2}\left(x_{n} ; n\right)$ remains a valid approximation for $\operatorname{Pr}_{\tau}\left[\ddot{X}_{n} \leq x_{n}\right]$ for all $x_{0} \geq 2$. In the process we derive a sharper approximation $\ddot{\pi}^{\mathrm{G} 23}\left(x_{n} ; n, x_{0}\right) \leq \ddot{\pi}^{\mathrm{G} 2}\left(x_{n} ; n\right)$ that depends on $x_{0}$ as well as $n$. The case $x_{0}=1$ is treated separately. 
Proposition 5.3. As in Proposition 5.2 let $Z_{n}=\ddot{X}_{n}-1, z_{0}=x_{0}-1, \theta=\tau$.

(i) Assume that $x_{0} \geq 2$, so $z_{0} \geq 1$. Then if $n$ is large and $z>0$,

$$
\operatorname{Pr}_{\tau}\left[Z_{n} \leq n z\right] \approx\left(1-\frac{2\left(z_{0}-1\right)}{n \sigma_{\tau}^{2}}\right) G_{2}\left(\frac{2 z}{\sigma_{\tau}^{2}}\right)+\frac{2\left(z_{0}-1\right)}{n \sigma_{\tau}^{2}} G_{3}\left(\frac{2 z}{\sigma_{\tau}^{2}}\right)
$$

so

$$
\begin{aligned}
& \operatorname{Pr}_{\tau}\left[\ddot{X}_{n} \leq x_{n}\right] \\
\approx & \left(1-\frac{2\left(x_{0}-2\right)}{n \sigma_{\tau}^{2}}\right) G_{2}\left(\frac{2 x_{n}}{n \sigma_{\tau}^{2}}\right)+\frac{2\left(x_{0}-2\right)}{n \sigma_{\tau}^{2}} G_{3}\left(\frac{2 x_{n}}{n \sigma_{\tau}^{2}}\right) \\
\equiv & \ddot{\pi}^{\mathrm{G} 23}\left(x_{n} ; n, x_{0}\right) .
\end{aligned}
$$

This reduces to (107) if $x_{0}=2$ or $n \sigma_{\tau}^{2} \gg 2\left(x_{0}-2\right)$.

(ii) Assume that $x_{0}=1$, so $z_{0}=0$, and define

$$
K=\min \left\{k \mid \ddot{X}_{k} \geq 2\right\}=\min \left\{k \mid Z_{k} \geq 1\right\} .
$$

Then if $n-K$ is large,

$$
\begin{aligned}
& \operatorname{Pr}_{\tau}\left[Z_{n} \leq(n-K) z \mid K, Z_{K}\right] \\
\approx & \left(1-\frac{2\left(Z_{K}-1\right)}{(n-K) \sigma_{\tau}^{2}}\right) G_{2}\left(\frac{2 z}{\sigma_{\tau}^{2}}\right)+\frac{2\left(Z_{K}-1\right)}{(n-K) \sigma_{\tau}^{2}} G_{3}\left(\frac{2 z}{\sigma_{\tau}^{2}}\right),
\end{aligned}
$$

so the conditional p-value given $K$ and $\ddot{X}_{K}$ can be approximated as follows:

$$
\begin{aligned}
& \operatorname{Pr}_{\tau}\left[\ddot{X}_{n} \leq x_{n} \mid K, \ddot{X}_{K}\right] \\
\approx & \left(1-\frac{2\left(\ddot{X}_{K}-2\right)}{(n-K) \sigma_{\tau}^{2}}\right) G_{2}\left(\frac{2 x_{n}}{(n-K) \sigma_{\tau}^{2}}\right)+\frac{2\left(\ddot{X}_{K}-2\right)}{(n-K) \sigma_{\tau}^{2}} G_{3}\left(\frac{2 x_{n}}{(n-K) \sigma_{\tau}^{2}}\right) \\
\equiv & \ddot{\pi}^{\mathrm{G} 23}\left(x_{n} ; n-K, \ddot{X}_{K}\right) .
\end{aligned}
$$

Proof. (i) First assume that $x_{0} \geq 3$, so $z_{0} \geq 2$. Rewrite (103) as follows. For $n=1$,

$$
Z_{1} \mid z_{0}=\left(\xi_{1}^{(1)}+W_{1}\right)+\left(\bar{\xi}_{1}^{(1)} \cdots+\bar{\xi}_{z_{0}-1}^{(1)}\right) \equiv U_{1}+V_{1}
$$

where the $\xi$ 's and $\bar{\xi}$ 's are i.i.d. copies of $\xi_{\tau}$. For $n \geq 2$,

$$
Z_{n} \mid Z_{n-1}=\left(\xi_{1}^{(n)}+\cdots+\xi_{U_{n-1}}^{(n)}+W_{n}\right)+\left(\bar{\xi}_{1}^{(n)} \cdots+\bar{\xi}_{V_{n-1}}^{(n)}\right) \equiv U_{n}+V_{n} .
$$


(If $V_{n-1}=0, V_{n}=0$.) Then $\left\{U_{n}\right\}$ is a critical GWI process with immigration rvs $\left\{W_{n}\right\}$ and initial size $u_{0}=1,\left\{V_{n}\right\}$ is a critical GW process with initial size $v_{0}=z_{0}-1=x_{0}-2 \geq 0$, and $\left\{U_{n}\right\}$ is independent of $\left\{V_{n}\right\}$. Therefore

$$
\begin{aligned}
& \operatorname{Pr}_{\tau}\left[2 Z_{n} \leq n \sigma_{\tau}^{2} z\right] \\
= & \operatorname{Pr}_{\tau}\left[2 U_{n} \leq n \sigma_{\tau}^{2} z\right] \operatorname{Pr}_{\tau}\left[V_{n}=0\right]+\operatorname{Pr}_{\tau}\left[2\left(U_{n}+V_{n}^{+}\right) \leq n \sigma_{\tau}^{2} z\right] \operatorname{Pr}_{\tau}\left[V_{n}>0\right] .
\end{aligned}
$$

Since $u_{0}=1$, Seneta's result applies to give $2 U_{n} / n \sigma_{\tau}^{2} \stackrel{d}{\rightarrow} G_{2}$, while by (92) $2 V_{n}^{+} / n \sigma_{\tau}^{2} \stackrel{d}{\rightarrow} G_{1}$. Because $U_{n}$ and $V_{n}$ are independent,

$$
\operatorname{Pr}_{\tau}\left[2\left(U_{n}+V_{n}^{+}\right) \leq n \sigma_{\tau}^{2} z\right] \rightarrow G_{3}(z) \text { as } n \rightarrow \infty .
$$

Furthermore, $n \operatorname{Pr}_{\tau}\left[V_{n}>0\right] \rightarrow 2\left(z_{0}-1\right) / \sigma_{\tau}^{2}$ by (93), so (115) yields (108), which, applying the continuity correction, yields (109) since $Z_{n}=\ddot{X}_{n}-1$.

If $x_{0}=2$ so $z_{0}=1$, then all $V_{n}=0$ and (108) reduces to Seneta's result for $\left\{U_{n}\right\}$.

(ii) The case $x_{0}=1$ differs because when $z_{0}=0$ the first nonzero value for the GWI process $\left\{Z_{n}\right\}$ is $Z_{K}=W_{K}$ and occurs when $n=K$. By conditioning on $K$ and $Z_{K}$ or $\ddot{X}_{K}$, however, (111) and (112) follow directly from (108) and (109) by replacing $z_{0}$ by $Z_{K}, x_{0}$ by $\ddot{X}_{K}$, and $n$ by $n-K$.

Like $\dot{\pi}^{\mathrm{EXP}}\left(x_{n} ; n\right)$, the approximate $p$-value $\ddot{\pi}^{\mathrm{G} 2}\left(x_{n} ; n\right)$ does not depend on $x_{0}(\geq 2)$; it conveys significance for $\dot{H}_{\geq}^{+}$(eventual extinction) iff $x_{n} \ll n \sigma_{\tau}^{2}$. We expect that $\ddot{\pi}^{\mathrm{G} 2}$, like $\dot{\pi}^{\mathrm{EXP}}$, will converge only slowly to the exact $p$ value, but that $\ddot{\pi}^{\mathrm{G} 23}$ will perform noticeably better. Note that $\ddot{\pi}^{\mathrm{G} 23}$ requires $n \sigma_{\tau}^{2}>2\left(x_{0}-2\right)$; otherwise the weight assigned to $G_{2}$ in (109) is negative.

Remark 5.2. The accuracy of the G2 and G23 approximations can be assessed for the geometric psod. The pmf of $\ddot{X}_{n}$ in the critical case can be obtained from (47) as follows: for $x_{n}>0$,

$$
\operatorname{Pr}_{\tau}\left[\ddot{X}_{n}=x_{n}\right]=\frac{x_{n}}{x_{0}} \operatorname{Pr}_{\tau}\left[X_{n}=x_{n}\right],
$$

and $\operatorname{Pr}_{\tau}\left[X_{n}=x_{n}\right]$ can be obtained explicitly as in Remark 5.1. Exact $p$-values and the G2 and G23 approximations are shown in Table 3, from which the superiority of G23 is apparent.

Remark 5.3. Moments of $\ddot{X}_{n}$ can be obtained from (106) by recursion, e.g.,

$$
\begin{aligned}
\mathrm{E}_{\tau}\left(\ddot{X}_{n}\right) & =x_{0}+n \sigma_{\tau}^{2}, \\
\operatorname{Var}_{\tau}\left(\ddot{X}_{n}\right) & =n\left[\omega_{\tau}+\left(\frac{n-3}{2}\right) \sigma_{\tau}^{4}+\left(x_{0}-3\right) \sigma_{\tau}^{2}-1\right],
\end{aligned}
$$

where $\omega_{\tau}=\mathrm{E}\left(\xi_{\tau}^{3}\right)$. 


\begin{tabular}{|lll|l|ll|lc|}
\hline & & & $x_{0}=2$ & \multicolumn{2}{|c|}{$x_{0}=8$} & \multicolumn{2}{c|}{$x_{0}=14$} \\
\hline$n$ & $x_{n}$ & $\ddot{\pi}^{G 2}$ & Exact & $\ddot{\pi}^{G 23}$ & Exact & $\ddot{\pi}^{G 23}$ & Exact \\
\hline 5 & 2 & 0.062 & 0.062 & & 0.022 & & 0.008 \\
5 & 4 & 0.191 & 0.169 & & 0.069 & & 0.028 \\
5 & 6 & 0.337 & 0.291 & & 0.134 & & 0.060 \\
\hline 10 & 4 & 0.062 & 0.063 & 0.029 & 0.038 & & 0.022 \\
10 & 8 & 0.191 & 0.180 & 0.105 & 0.115 & & 0.073 \\
10 & 12 & 0.337 & 0.313 & 0.207 & 0.213 & & 0.143 \\
\hline 20 & 8 & 0.062 & 0.063 & 0.045 & 0.048 & 0.029 & 0.037 \\
20 & 12 & 0.122 & 0.120 & 0.092 & 0.094 & 0.063 & 0.074 \\
20 & 16 & 0.191 & 0.186 & 0.148 & 0.148 & 0.105 & 0.118 \\
\hline 50 & 16 & 0.041 & 0.042 & 0.037 & 0.038 & 0.033 & 0.034 \\
50 & 24 & 0.084 & 0.084 & 0.076 & 0.076 & 0.067 & 0.069 \\
50 & 32 & 0.135 & 0.134 & 0.122 & 0.122 & 0.109 & 0.111 \\
\hline
\end{tabular}

Table 3: Exact and approximate $p$-values $\operatorname{Pr}_{\tau}\left[\ddot{X}_{n}^{+} \leq x_{n}\right]$ for the geometric psod when $x_{0}=2,8,14$.

\section{Predicting extinction or explosion: sequential sampling}

6.1. Sequential probability ratio tests (SPRT). The SPRT (Barnard (1946), Wald (1947), Ghosh (1970), Stuart and Ord (1991)) is well suited for the following sequential version of the prediction problem:

Based on sequential data $\mathbf{x} \equiv\left(x_{0}, x_{1}, x_{2}, \ldots\right)$ from a psod $G W$ process $\mathbf{X} \equiv$ $\mathbf{X}_{\theta}$ with initial size $x_{0}$, predict whether extinction or explosion will occur for the current realization of the process.

Unlike Section 5, non-termination need not be assumed. This prediction problem can be formulated as the following testing problem:

Based on observing $\mathbf{X}$ sequentially, test

$$
\begin{aligned}
& \dot{H}_{\geq}: \mathbf{X} \stackrel{d}{=} \dot{\mathbf{X}}_{\theta}, \theta \geq \tau(\text { eventual extinction }) \\
\text { vs. } & \ddot{H}_{\geq}: \mathbf{X} \stackrel{d}{=} \ddot{\mathbf{X}}_{\theta}, \theta \geq \tau(\text { eventual explosion }) .
\end{aligned}
$$

For fixed $\theta \geq \tau$, the SPRT for testing $\dot{f}_{\theta}$ vs. $\ddot{f}_{\theta}$ has the following form: 
The $\operatorname{SPRT}(\theta ; B, A)$ : fix $0<B<1<A<\infty$. For $n=1,2, \ldots$,

$$
\begin{cases}\text { stop and accept } \dot{H}_{\geq} \text {(predict extinction) } & \text { if } \lambda_{\theta}\left(\mathbf{x}_{n}\right) \leq B, \\ \text { stop and accept } \ddot{H}_{\geq} \text {(predict explosion) } & \text { if } \lambda_{\theta}\left(\mathbf{x}_{n}\right) \geq A, \\ \text { continue sampling } & \text { if } B<\lambda_{\tau}\left(\mathbf{x}_{n}\right)<A,\end{cases}
$$

where $\lambda_{\theta}\left(\mathbf{x}_{n}\right)=\ddot{f}_{\theta}\left(\mathbf{x}_{n}\right) / \dot{f}_{\theta}\left(\mathbf{x}_{n}\right)$.

The stopping time for the $\operatorname{SPRT}(\theta ; B, A)$ is a random variable $N(\theta ; B, A)$. Because $\operatorname{Pr}_{\theta^{\prime}}\left[X_{n} \rightarrow 0\right.$ or $\left.\infty\right]=1$ for all $\theta^{\prime} \geq \tau, N(\theta ; B, A)$ is finite with probability 1. As $B$ decreases and $A$ increases, $\mathrm{E}_{\theta^{\prime}}[N(\theta ; B, A)]$ increases under both $\dot{H}_{\geq}$and $\ddot{H}_{\geq}$, but the error probabilities $\alpha_{\theta^{\prime}} \equiv \alpha_{\theta^{\prime}}(\theta ; B, A)$ and $\beta_{\theta^{\prime}} \equiv \beta_{\theta^{\prime}}(\theta ; B, A)$ need not both decrease (cf. Wald (1947, p.45)). Here,

$$
\begin{aligned}
\alpha_{\theta^{\prime}}(\theta ; B, A) & \equiv \operatorname{Pr}_{\theta^{\prime}}\left[\operatorname{SPRT}(\theta ; B, A) \text { accepts } \ddot{H}_{\geq} \mid \dot{H}_{\geq}\right] \\
\beta_{\theta^{\prime}}(\theta ; B, A) & \equiv \operatorname{Pr}_{\theta^{\prime}}\left[\operatorname{SPRT}(\theta ; B, A) \text { accepts } \dot{H}_{\geq} \mid \ddot{H}_{\geq}\right]
\end{aligned}
$$

Wald (1947, §3.2) derived the following upper bounds: for any $\theta \geq \tau$,

$$
\begin{aligned}
\alpha_{\theta}(\theta ; B, A) & \leq \frac{1-\beta_{\theta}(\theta ; B, A)}{A} \leq \frac{1}{A}, \\
\beta_{\theta}(\theta ; B, A) \leq\left(1-\alpha_{\theta}(\theta ; B, A)\right) B & \leq B .
\end{aligned}
$$

Thus if $\alpha$ and $\beta$ are prespecified, we may choose $B=\beta$ and $A=\frac{1}{\alpha}$ to guarantee that $\operatorname{SPRT}\left(\theta ; \beta, \frac{1}{\alpha}\right)$ satisfies the error bounds

$$
\alpha_{\theta}\left(\theta ; \beta, \frac{1}{\alpha}\right) \leq \alpha \quad \text { and } \quad \beta_{\theta}\left(\theta ; \beta, \frac{1}{\alpha}\right) \leq \beta .
$$

Wald also derived the following approximations: if $\alpha+\beta<1$ then $\operatorname{SPRT}\left(\theta ; \frac{\beta}{1-\alpha}, \frac{1-\beta}{\alpha}\right)$ more nearly attains the specified error probabilities $\alpha$ and $\beta$ than does $\operatorname{SPRT}\left(\theta ; \beta, \frac{1}{\alpha},\right)$, i.e.,

$$
\begin{aligned}
& \alpha_{\theta}\left(\theta ; \frac{\beta}{1-\alpha}, \frac{1-\beta}{\alpha}\right) \approx \alpha, \quad \beta_{\theta}\left(\theta \frac{\beta}{1-\alpha}, \frac{1-\beta}{\alpha}\right) \approx \beta, \\
& \alpha_{\theta}\left(\theta ; \frac{\beta}{1-\alpha}, \frac{1-\beta}{\alpha}\right)+\beta_{\theta}\left(\theta ; \frac{\beta}{1-\alpha}, \frac{1-\beta}{\alpha}\right) \leq \alpha+\beta .
\end{aligned}
$$

6.2. The least favorable distribution for sequential sampling. Because $\theta$ is unknown, the $\operatorname{SPRT}(\theta ; \cdot, \cdot)$ cannot be applied directly (but see Section 6.3.) When the psod satisfies TP2a and TP2b, however, like (81) the testing problem (120) has a convenient solution, namely the $\operatorname{SPRT}(\tau ; \cdot, \cdot)$. 
Propositions 4.1 and 4.2 imply that $\dot{H}_{\geq}$and $\ddot{H}_{\geq}$are separated families and that $\left(\dot{f}_{\tau}, \ddot{f}_{\tau}\right)$ is a pair of least favorable distributions for $(120)$. Furthermore, by Propositions 4.1 and 4.2, $\alpha_{\theta^{\prime}}$ and $\beta_{\theta^{\prime}}$ both decrease as $\theta^{\prime}$ increases. Therefore $\operatorname{SPRT}\left(\tau ; \beta, \frac{1}{\alpha}\right)$ (respectively, $\operatorname{SPRT}\left(\tau ; \frac{\beta}{1-\alpha}, \frac{1-\beta}{\alpha}\right)$ ) is an optimal test for $\dot{f}_{\tau}$ vs. $\ddot{f}_{\tau}$ for which $\alpha_{\theta^{\prime}} \leq \alpha$ and $\beta_{\theta^{\prime}} \leq \beta$ (resp., approximately) for all $\theta^{\prime} \geq \tau$. Specifically, by (41) and (47),

$$
\lambda_{\tau}\left(\mathbf{x}_{n}\right) \equiv \frac{\ddot{f}_{\tau}\left(\mathbf{x}_{n}\right)}{\dot{f}_{\tau}\left(\mathbf{x}_{n}\right)}=\frac{x_{n}}{x_{0}},
$$

so the $\operatorname{SPRT}(\tau ; B, A)$ assumes the simple form

$$
\begin{cases}\text { stop and accept } \dot{H}_{\geq} \text {(predict extinction) } & \text { if } x_{n} \leq x_{0} B, \\ \text { stop and accept } \ddot{H}_{\geq} \text {(predict explosion) } & \text { if } x_{n} \geq x_{0} A, \\ \text { continue sampling } & \text { if } x_{0} B<x_{n}<x_{0} A .\end{cases}
$$

Note that this is a universal prediction procedure, that is, it is valid for any psod, in particular it does not depend on $\sigma_{\tau}^{2}$. As a consequence, however, it is somewhat conservative.

6.3. A less conservative sequential prediction procedure. If $\theta$ were known $(\theta>\tau)$, the $\operatorname{SPRT}\left(\theta ; \beta, \frac{1}{\alpha}\right)$ (respectively, $\left.\operatorname{SPRT}\left(\theta ; \frac{\beta}{1-\alpha}, \frac{1-\beta}{\alpha}\right)\right)$ provides an optimal test for $\dot{f}_{\theta}$ vs. $\ddot{f}_{\theta}$ for which $\alpha_{\theta} \leq \alpha$ and $\beta_{\theta} \leq \beta$ (resp., $\alpha_{\theta} \approx \alpha$ and $\beta_{\theta} \approx \beta$ ). From (12) and (13),

$$
\lambda_{\theta}\left(\mathbf{x}_{n}\right) \equiv \frac{\ddot{f}_{\theta}\left(\mathbf{x}_{n}\right)}{\dot{f}_{\theta}\left(\mathbf{x}_{n}\right)}=\frac{u_{\theta}^{-x_{n}}-1}{u_{\theta}^{-x_{0}}-1} .
$$

Because $\lambda_{\theta}\left(\mathbf{x}_{n}\right)$ is strictly increasing in $x_{n}$, the $\operatorname{SPRT}(\theta ; B, A)$ is given by

$$
\begin{cases}\text { stop and accept } \dot{H}_{\theta} \text { (predict extinction) } & \text { if } X_{n} \leq x_{0} \ell\left(u_{\theta}^{x_{0}}, B\right), \\ \text { stop and accept } \ddot{H}_{\theta} \text { (predict explosion) } & \text { if } X_{n} \geq x_{0} \ell\left(u_{\theta}^{x_{0}}, A\right), \\ \text { continue sampling } & \text { if } x_{0} \ell\left(u_{\theta}^{x_{0}}, B\right)<X_{n}<x_{0} \ell\left(u_{\theta}^{x_{0}}, A\right),\end{cases}
$$

where

$$
\ell(u, \eta)=\frac{\log \left[\left(\frac{1-u}{u}\right) \eta+1\right]}{\log \left(\frac{1}{u}\right)}, \quad 0<u<1, \quad 0 \leq \eta<\infty .
$$

For fixed $u, \ell(u, \eta)$ increases strictly and continuously from 0 to $\infty$ as $\eta$ ranges from 0 to $\infty$; also $\ell(u, 0)=0$ and $\ell(u, 1)=1$. Define $\ell(1, \eta)=\ell(1-, \eta)=\eta$. 
Lemma 6.1. If $0<u<1$ and $0<\eta<1$ (resp., $1<\eta<\infty)$, then $\ell(u, \eta)$ is strictly decreasing (resp., strictly increasing) in $u$ and

$$
\eta<\ell(u, \eta)<1 \quad(\text { resp., } 1<\ell(u, \eta)<\eta) .
$$

Proof. Set $v=\frac{1-u}{u}$, so that $0<v<\infty$ and

$$
\ell(u, \eta)=\frac{\log [v \eta+1)]}{\log (v+1)} \equiv \bar{\ell}(v, \eta) .
$$

For $1<\eta<\infty$, to show that $\ell(u, \eta)$ is strictly increasing for $0<u<1$, it suffices to show that $\bar{\ell}(v, \eta)$ is strictly decreasing for $0<v<\infty$, that is, $\partial \bar{\ell}(v, \eta) / \partial v<0$. This is equivalent to showing that

$$
\frac{\eta \log (v+1)}{\eta v+1}-\frac{\log (\eta v+1)}{v+1}<0,
$$

equivalently, that

$$
\Delta(v, \eta) \equiv(v+1) \log (v+1)-\left(v+\frac{1}{\eta}\right) \log (\eta v+1)<0 .
$$

But $\Delta(v, 1)=0$ for $\eta=1$ and

$$
\begin{aligned}
\frac{\partial \Delta(v, \eta)}{\partial \eta} & =-\frac{\left(v+\frac{1}{\eta}\right) v}{\eta v+1}+\frac{\log (\eta v+1)}{\eta^{2}} \\
& =-\frac{1}{\eta^{2}}[\eta v-\log (\eta v+1)]<0,
\end{aligned}
$$

hence (A.1) holds. Then by L'Hospital's rule,

$$
\eta=\bar{\ell}(0+, \eta)>\bar{\ell}(v, \eta)>\bar{\ell}(\infty-, \eta)=1,
$$

which yields the desired inequalities for $\ell(u, \eta)$. The results for $0<\eta<1$ are established in similar fashion.

From Lemma 6.1, the lower (resp., upper) stopping boundary for the $\operatorname{SPRT}(\theta ; B, A)$ strictly increases (resp., strictly decreases) as $\theta$ increases on $[\tau, \psi)$, hence the stopping region decreases and $N(\theta ; B, A)$ decreases. Thus

$$
x_{0} B<x_{0} \ell\left(u_{\theta}^{x_{0}}, B\right)<x_{0}<x_{0} \ell\left(u_{\theta}^{x_{0}}, A\right)<x_{0} A .
$$


This difference can be substantial (see Table 5) and implies that

$$
\mathrm{E}_{\theta^{\prime}}[N(\theta ; B, A)]<\mathrm{E}_{\theta^{\prime}}[N(\tau ; B, A)] \text { for all } \theta^{\prime} \geq \tau .
$$

Thus if one is willing to assume a fairly unrestrictive upper bound $u_{\theta} \leq$ $\bar{u}<1$ for the extinction probability $u_{\theta}$ (e.g., $\bar{u}=0.90,0.95$, or 0.99 ), corresponding to an unrestrictive lower bound $\theta \geq \underline{\theta} \equiv \theta_{\bar{u}}>\tau$ for $\theta$ itself, then by using the $\operatorname{SPRT}\left(\underline{\theta} ; \beta, \frac{1}{\alpha}\right)$ or $\left.\operatorname{SPRT}\left(\underline{\theta} ; \frac{\beta}{1-\alpha}, \frac{1-\beta}{\alpha}\right)\right)$, by Proposition 4.1(ii) one would control the first error probability for problem (120), i.e., $\alpha_{\theta} \leq \alpha$ for all $\theta \geq \underline{\theta}$, while substantially reducing the expected stopping time. If TP2a and TP2b hold, then by Proposition 4.2(iii) the second error probability also would be controlled, i.e., $\beta_{\theta} \leq \beta$ for all $\theta \geq \underline{\theta}$.

Remark 6.1. For the Poisson $(\theta)$ psod, it follows from (33) that

$$
\theta_{\bar{u}}=-\frac{\log (\bar{u})}{1-\bar{u}}
$$

so $\theta_{.90}=1.0536, \theta_{.95}=1.0259, \theta_{.99}=1.0050$, which lower bounds are close to the critical value $\tau=1$. For the negative $\operatorname{binomial}(r, \theta) \operatorname{psod}$, (35) yields

$$
\theta_{\bar{u}}=\frac{1-\bar{u}^{\frac{1}{r}}}{1-\bar{u}^{\frac{r+1}{r}}}
$$

which reduces to $\theta_{\bar{u}}=\frac{1}{1+\bar{u}}$ for the geometric $(\theta)$ psod when $r=1$. Here again this lower bound will be close to the critical value $\tau=\frac{1}{1+r}$ if $\bar{u}$ is close to 1 . In these cases, therefore, the assumption that $u_{\theta} \leq \bar{u}$ is not very restrictive for $\bar{u}=.90, .95, .99$.

Remark 6.2. Unlike the fixed- $n$ prediction procedures derived in $\S 5$ (cf. Remarks 5.1 and 5.2), the SPRTs compare $x_{n}$ to $x_{0}$ rather than to $n$ in order to predict whether the observed process is on a trajectory toward extinction or toward explosion. Note that if $x_{0}$ is small, the SPRTs are useful for predicting explosion but not for predicting extinction. For example,

$$
x_{0} \ell\left(u_{\theta}^{x_{0}}, \beta\right)<1 \Longleftrightarrow x_{0}<\ell\left(u_{\theta}, \frac{1}{\beta}\right),
$$

so if $x_{0}<\ell\left(u_{\theta}, \frac{1}{\beta}\right)$ then the $\operatorname{SPRT}\left(\theta ; \beta, \frac{1}{\alpha}\right)$ reduces to the $\operatorname{SPRT}\left(\theta ; 0, \frac{1}{\alpha}\right)$ :

$$
\begin{cases}\text { stop and accept } \dot{H}_{\theta} \text { (predict extinction) } & \text { if } X_{n}=0, \\ \text { stop and accept } \ddot{H}_{\theta} \text { (predict explosion) } & \text { if } X_{n} \geq x_{0} \ell\left(u_{\theta}^{x_{0}}, \frac{1}{\alpha}\right), \\ \text { continue sampling } & \text { if } 1 \leq X_{n}<x_{0} \ell\left(u_{\theta}^{x_{0}}, \frac{1}{\alpha}\right),\end{cases}
$$


hence will predict extinction only if extinction actually occurs. If $x_{0}<\frac{1}{\beta}$, the $\operatorname{SPRT}\left(\tau ; \beta, \frac{1}{\alpha}\right)$ also reduces to $\operatorname{SPRT}\left(\theta ; 0, \frac{1}{\alpha}\right)$ hence behaves similarly.

Remark 6.3. Note that the $\operatorname{SPRT}(\theta ; B, A)$ depends on $\theta$ only through the value of the extinction probability $u_{\theta}$, not on the specific offspring distribution, whether a psod or not. Therefore, hereafter we shall use the notation $\operatorname{SPRT}\left(u_{\theta} ; B, A\right)$, or simply $\operatorname{SPRT}(u ; B, A)$. In particular, the universal $\operatorname{SPRT}(\tau ; B, A)$ in $\S 9.2$ is now designated as $\operatorname{SPRT}(1 ; B, A)$.

\section{Examples}

Four examples are presented to illustrate the fixed- $n(\S 5)$ and sequential $(\S 6)$ procedures for predicting extinction or explosion from the current realization of a GW process. Because the Poisson, negative binomial, and geometric psods are assumed, conditions TP2a and TP2b are satisfied, so these prediction procedures possess the properties asserted in $\S 5.2,6.2$, and 6.3.

Example 7.1: Smallpox in Sao Paolo, Brazil. An outbreak of variola minor in Sao Paolo occurred in 1956 (see Table 4). This outbreak was caused by a single infectious individual and lasted four generations before the schools closed; see Becker (1972), Guttorp (1991, p.59). Becker (1977) and Heyde (1979) modeled these data by a GW process; also see Guttorp (1991, p.58). Like Heyde we assume a Poisson $(\theta)$ psod; here $\mu_{\theta}=\theta, \tau=1, \sigma_{\tau}^{2}=1$.

\begin{tabular}{|c|ccccc|}
\hline$n$ & 0 & 1 & 2 & 3 & 4 \\
\hline$x_{n}$ & 1 & 5 & 3 & 12 & 24 \\
\hline
\end{tabular}

Table 4: Occurrences of variola minor in Sao Paolo, Brazil, 1956.

These data suggest a trajectory toward explosion. To assess the strength of this prediction, first consider the fixed- $n$ testing problem (81) with $\dot{H}_{\geq}^{+}$ (eventual extinction) taken to be the null hypothesis and $\ddot{H}_{\geq}^{+}$(explosion) the alternative. Here $x_{0}=1, n=4, x_{n}=24$ so the exponential approximation (101) for the $p$-value of the fixed- $n$ prediction procedure (87) is

$$
\dot{\pi}^{\mathrm{EXP}}(24 ; 4)=e^{-\frac{47}{4}} \approx 7.89 \times 10^{-6}
$$

which strongly supports the prediction of explosion. Because $n=4$ is not large, this approximation is not entirely reliable. (Because $x_{0}=1$, the EXP and GAM approximations coincide.) 


\begin{tabular}{|c|r|cc|cc|}
\hline$x_{0}$ & $\bar{u}$ & $x_{0} \ell\left(\bar{u}^{x_{0}}, 0.05\right)$ & $x_{0} \ell\left(\bar{u}^{x_{0}}, \frac{1}{0.05}\right)$ & $x_{0} \ell\left(\bar{u}^{x_{0}}, 0.01\right)$ & $x_{0} \ell\left(\bar{u}^{x_{0}}, \frac{1}{0.01}\right)$ \\
\hline 1 & 1.00 & 0.05 & 20 & 0.01 & 100 \\
& 0.99 & 0.05 & 18.3 & 0.01 & 69.5 \\
& 0.95 & 0.05 & 14.0 & 0.01 & 35.8 \\
& 0.90 & 0.05 & 11.1 & 0.01 & 23.7 \\
\hline 14 & 1.00 & 0.70 & 280 & 0.14 & 1400 \\
& 0.99 & 0.75 & 138.5 & 0.15 & 276.5 \\
& 0.95 & 0.99 & 60.3 & 0.20 & 90.9 \\
& 0.90 & 1.48 & 40.1 & 0.31 & 55.3 \\
\hline 38 & 1.00 & 1.90 & 760 & 0.38 & 3800 \\
& 0.99 & 2.29 & 232.1 & 0.46 & 384.2 \\
& 0.95 & 5.13 & 93.6 & 1.14 & 124.8 \\
& 0.9 & 12.39 & 66.3 & 4.09 & 81.5 \\
\hline
\end{tabular}

Table 5: Stopping boundaries for $\operatorname{SPRT}\left(\bar{u} ; \beta, \frac{1}{\alpha}\right), \alpha=\beta=0.05$ and 0.01 .

Next we apply the sequential testing approach. Here $x_{0}=1$, so the stopping boundaries for the sequential prediction procedure $\operatorname{SPRT}\left(\bar{u} ; \beta, \frac{1}{\alpha}\right)$ (cf. Remark 6.3) appear in the first tier of Table 5 for $\alpha=\beta=.05, .01$ and $\bar{u}=1.0, .99, .95,0.90$. As $\bar{u}$ decreases, $\operatorname{SPRT}\left(\bar{u} ; \beta, \frac{1}{\alpha}\right)$ becomes less conservative, stopping more quickly. For example, $\operatorname{SPRT}\left(\bar{u}=1.0 ; .05, \frac{1}{.05}\right)$ stops and predicts explosion when $x_{n} \geq 20$, which here occurs when $n=4$, while $\operatorname{SPRT}\left(\bar{u}=.90 ; .05, \frac{1}{05}\right)$ stops and predicts explosion when $x_{n} \geq 11.1$, which occurs when $n=3$.

The $\operatorname{SPRT}\left(\bar{u}=.90 ; .05, \frac{1}{.05}\right)$ requires the assumption that $u_{\theta} \leq \bar{u}=.90$, equivalently $\theta \geq \theta_{\bar{u}}=1.0536$, see Remark 6.1 . The reliability of this assumption can be assessed in two ways. First, $y_{n-1}=21$ and $y_{n}=45$ so $\hat{\theta}=\hat{\mu}=(45-1) / 21 \approx 2.095$ from $(77)$, which is substantially larger than 1.0536. Second, an estimate of $u_{\theta}$ could be obtained from the nonparametric MLE $\hat{\mathbf{p}}$ of the offspring distribution $\mathbf{p}_{\theta}$ (cf. Guttorp (1991, Proposition 3.4), also Stigler (1971)), but this would require knowledge of the family histories of each infected individual, which is unavailable. Here, however, $\hat{\mathbf{p}}$ can be obtained from the EM algorithm because $n$ is small, cf. Guttorp (1991, pp. 119-120). For $n=3, \hat{\mathbf{p}}$ puts masses $(0.239,0.428,0.206,0.127)$ on $0,1,5$, 6 , and from (3) the estimated extinction probability for this distribution is 0.424. For $n=4$ the estimated distribution puts masses $(0.332,0.147,0.219$, 
0.302 ) on $0,1,2$, and 5 , yielding an estimated extinction probability 0.447 . Both estimates fall well below the assumed upper bound $\bar{u}=0.9$.

Because the outbreak terminated at the 7 th generation, fixed- $n$ prediction methods $(\S 5)$ are not relevant. Instead, beause $x_{0}=1$ the stopping boundaries of $\operatorname{SPRT}\left(\bar{u} ; \beta, \frac{1}{\alpha}\right)$ for $\bar{u}=1.0,0.99,0.95,0.90$ and $\alpha=\beta=.05, .01$ again appear in the first tier of Table 5 . Because $1 \leq x_{n} \leq 7$ for $n=1, \ldots, 6$ in this example, none of these SPRTs would stop sampling until the extinction observed at $n=7$. Note that $\hat{\theta}=\hat{\mu}=(30-1) / 30=0.967<1$ by $(77)$ $\left(y_{n-1}=y_{n}=30\right)$.

Example 7.2: Pertussis in Washington State, 2012. The weekly number of new cases of pertussis remained fairly constant in 2011 (Figure 1) but increased dramatically at the beginning of 2012 (Table 6), suggesting possible explosion. Here $x_{0}=1, n=11, x_{n}=98, y_{n-1}=594$, and $y_{n}=692$. The MLE $\hat{\mu}=691 / 594=1.1633$ and $\tilde{\sigma}^{2}=8.0342$, where

$$
\tilde{\sigma}^{2} \equiv \frac{1}{n} \sum_{\nu=1}^{n} x_{\nu-1}\left(\frac{x_{\nu}}{x_{\nu-1}}-\hat{\mu}\right)^{2}
$$

is Dion's (1975) estimate of the offspring variance, cf. Guttorp (1991, p.109).

Because $\hat{\mu} \ll \tilde{\sigma}^{2}$, the Poisson distribution does not fit these data. Instead, since $\hat{\mu}$ and $\tilde{\sigma}^{2}$ agree with the mean and variance of the negative binomial $\mathrm{NB}(\hat{r}, \hat{\theta})$ psod with $\hat{r}=0.1970$ and $\hat{\theta}=0.8552$ (cf. Example 3.2), we shall assume the model $\mathrm{NB}(r=0.1970, \theta)$ with $\theta$ unknown $(0<\theta<1)$, so $\tau=r(1+r)^{-1}=0.1646$ and $\sigma_{\tau}^{2}=6.0761$.

\begin{tabular}{|c|cccccccccccc|}
\hline Week & 1 & 2 & 3 & 4 & 5 & 6 & 7 & 8 & 9 & 10 & 11 & 12 \\
\hline$n$ & 0 & 1 & 2 & 3 & 4 & 5 & 6 & 7 & 8 & 9 & 10 & 11 \\
\hline$x_{n}$ & 1 & 7 & 22 & 38 & 50 & 65 & 78 & 61 & 74 & 96 & 102 & 98 \\
\hline
\end{tabular}

Table 6: Weekly occurrences of pertussis in Washington State, 2012.

To assess the evidence for a prediction of explosion, first consider the fixed- $n$ testing problem (81) with $\dot{H}_{\geq}^{+}$(eventual extinction) taken to be the null hypothesis and $\ddot{H}_{\geq}^{+}$(eventual explosion) the alternative. From (101) the exponential approximation to the $p$-value of the fixed- $n$ procedure (87) is

$$
\dot{\pi}^{\mathrm{EXP}}(98 ; 11)=e^{-\frac{195}{11(6.0761)}} \approx 0.054
$$


which moderately supports the prediction of explosion.

By contrast, from Tables 5 and 6 the conservative $\operatorname{SPRT}\left(1.0 ; \beta, \frac{1}{\alpha}\right)$ with $\alpha=\beta=0.05$ would have stopped and predicted explosion by Week 3 ! With $\alpha=\beta=0.01$ this SPRT would not have stopped until Week 11, but the $\operatorname{SPRT}\left(0.90 ; 0.01, \frac{1}{0.01}\right)$ would have predicted explosion by Week 4 .

In fact a state of health emergency was declared after Week 14 and an

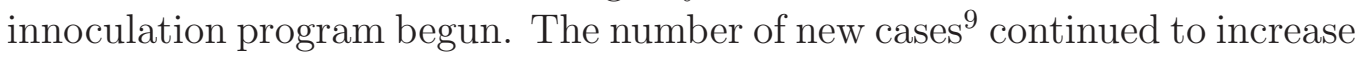
to a peak of 254 in Week 20, then declined to 23 new cases in Week 52. Had these sequential prediction procedures been applied, this program could have begun much earlier, possibly greatly reducing the total number of cases.

We note that the prediction for 2012 (explosion) is the opposite of that which our methods would obtain for 2011 (extinction), even though the increase in $\hat{\mu}$ from 2011 to 2012 is small, namely 1.0455 vs. 1.1633 .

Example 7.3: California condors. Wilbur (1978) gives the annual population counts of the threatened California condor from 1968 through 1976 (see Table 7). Here $x_{0}=38, n=8, x_{n}=19, y_{n-1}=183$, and $y_{n}=202$; the MLE $\hat{\mu}=164 / 183=0.8962$ and $\tilde{\sigma}^{2}=2.2755$. Because $\tilde{\sigma}^{2}$ is not greatly different from the estimated variance $\sigma_{\hat{\theta}}^{2}=\hat{\theta} /(1-\hat{\theta})^{2}=1.6992$ under the geometric $\operatorname{GM}(\hat{\theta})$ distribution with $\hat{\theta}=\hat{\mu} /(1+\hat{\mu})=.4726$ (see Example 3.2), we will assume the $\operatorname{GM}(\theta)$ psod model $(0<\theta<1)$ to illustrate its ease of application. For this model $A(\theta)=1 /(1-\theta), \tau=1 / 2$, and $\sigma_{\tau}^{2}=2$.

\begin{tabular}{|c|ccccccccc|}
\hline Year & 1968 & 1969 & 1970 & 1971 & 1972 & 1973 & 1974 & 1975 & 1976 \\
\hline$n$ & 0 & 1 & 2 & 3 & 4 & 5 & 6 & 7 & 8 \\
\hline Count $x_{n}$ & 38 & 26 & 27 & 18 & 25 & 19 & 19 & 11 & 19 \\
\hline
\end{tabular}

Table 7: Annual counts of California condors 1968-1976.

The data in Table 7 suggest a declining population, hence possible extinction. To evaluate this prediction, first consider the fixed- $n$ testing problem (81) with $\ddot{H}_{>}^{+}$(eventual explosion) as the null hypothesis and $\dot{H}_{>}^{+}$(eventual extinction) the alternative. Here $x_{0}=38$ and $n=8$ so $n \sigma_{\tau}^{2}<2\left(x_{0}-2\right)$, hence the approximations $\ddot{\pi}^{\mathrm{G} 2}$ in (107) and $\ddot{\pi}^{\mathrm{G} 23}$ in (109) for the fixed- $n$ prediction procedures (87) are inapplicable (cf. Proposition 5.3(i)).

\footnotetext{
${ }^{9}$ The weekly data shown have since been revised. We have used the unrevised data because it was those upon which public health decisions were based.
} 
By contrast, the sequential prediction procedure $\operatorname{SPRT}\left(0.9 ; 0.05, \frac{1}{0.05}\right)$ would have stopped in 1975 and predicted extinction. (Compare the data in Table 7 to the stopping boundaries in the last row of Table 5.)

In fact, by the mid 1980's all remaining wild condors were captured and moved to zoos, where a breeding program was begun, followed by relocation back to the wild. By 2011 the total wild population had grown to 191, in addition to 178 remaining in captivity.

Example 7.4: North American whooping cranes. Miller et al. (1974) give the annual counts of migrating whooping cranes, an endangered species, arriving in Texas from $1938(n=0)$ through $1972(n=34)$; see Figure 4 and Guttorp (1991, p.190)). Here $x_{0}=14, n=34, x_{n}=51, y_{n-1}=1072$, and $y_{n}=1123$; the MLE $\hat{\mu}=1109 / 1072=1.0345$. Since $\hat{\mu}$ does not differ greatly from Dion's estimate $\tilde{\sigma}^{2}=0.84$, the Poisson $(\theta)$ psod model is assumed.

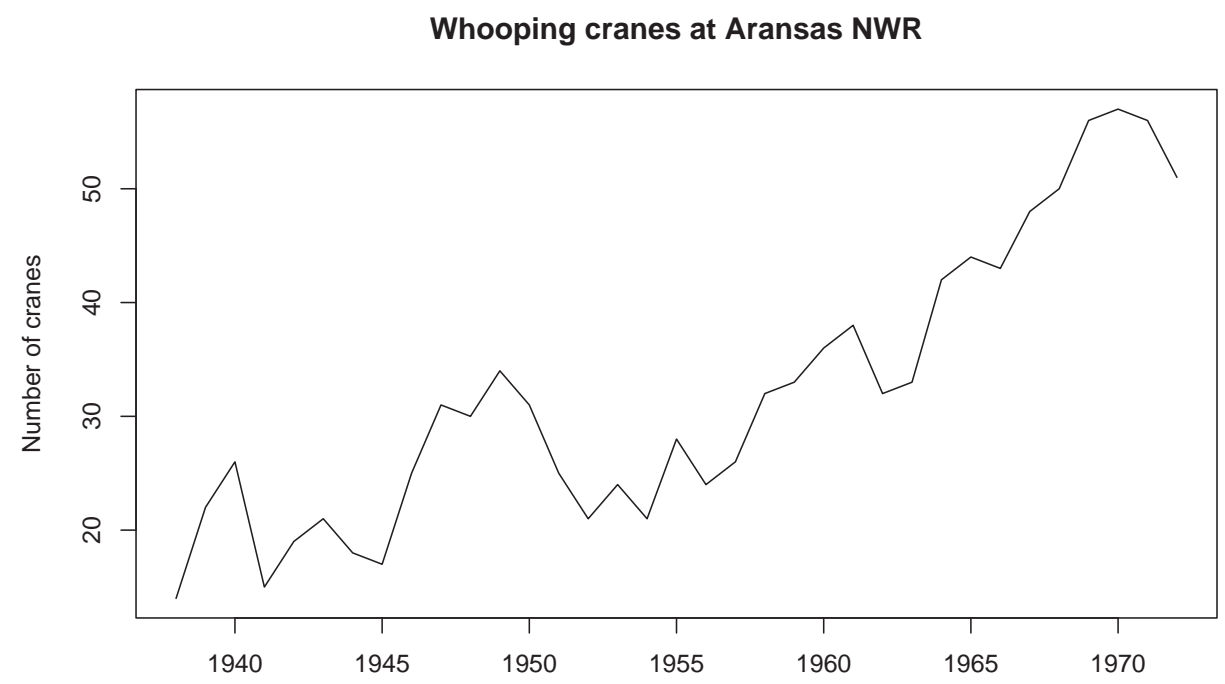

Figure 4: North American whooping crane population counts 1938-1972.

The counts in Figure 4 show an increasing trend, suggesting explosion. To evaluate this prediction, first consider the fixed- $n$ testing problem (81) with $\dot{H}_{\geq}^{+}$(eventual extinction) as the null hypothesis and $\ddot{H}_{\geq}^{+}$(eventual explosion) as the alternative. Here $\sigma_{\tau}^{2}=1$, so the EXP and GAM approximations (101) 
and (102) for the $p$-values of the fixed- $n$ prediction procedure (87) are

$$
\begin{aligned}
\dot{\pi}^{\operatorname{EXP}}(51 ; 34) & =e^{-\frac{101}{34}} \approx 0.051 \\
\dot{\pi}^{\mathrm{GAM}}(51 ; 34,14) & =\frac{17}{14}\left(\frac{16}{17}\right)^{14} \sum_{r=1}^{14}\left(\begin{array}{c}
14 \\
r
\end{array}\right) \frac{1}{16^{r}} \bar{G}_{r}\left(\frac{101}{34}\right) \approx 0.086
\end{aligned}
$$

respectively, with $\dot{\pi}^{\mathrm{GAM}}$ expected to be more accurate. This provides modest support for a prediction of explosion.

By contrast, the sequential prediction procedure $\operatorname{SPRT}\left(0.9 ; 0.05, \frac{1}{0.05}\right)$ would have stopped in $1964\left(n=26, x_{n}=42>40.1\right)$ and predicted explosion, while $\operatorname{SPRT}\left(0.9 ; 0.01, \frac{1}{0.01}\right)$ would have stopped in $1969\left(n=31, x_{n}=56>55.3\right)$ and predicted explosion. (The values 40.1 and 55.3 appear in the second tier of Table 5.)

Acknowledgement: We are grateful to Brayan Ortiz for his assistance with numerical computations.

\section{References}

Athreya, K. B. and Ney, P. E. (1972). Branching Processes. Springer-Verlag, Berlin.

Barnard, G. A. (1946). Sequential tests in industrial statistics (with discussion). J. Royal Statist. Soc. Supplement 8, 1-26.

Becker, N. (1972). Vaccination programmes for rare infectious diseases. Biometrika 59 443-453.

Becker, N. (1974). On parametric estimation for mortal branching processes. Biometrika 61 393-399.

Becker, N. (1977). Estimation for discrete time branching processes with application to epidemics. Biometrics 33 515-522.

Centers for Disease Control and Prevention (2012). Pertussis epidemic Washington, 2012; Morbidity and Mortality Weekly Report. Online at: http://www.cdc.gov/mmwr/preview/mmwrhtml/mm6128a1.htm

Dion, J.-P. (1975). Estimation of the variance of a branching process. Ann. Statist. 3 1183-1187.

Feller, W. (1968). An Introduction to Probability Theory and its Applications, Third Edition. Wiley, NY. 
Ghosh, B. K. (1970). Sequential Tests of Statistical Hypotheses. AddisonWesley, Reading, PA.

Guttorp, P. (1991). Statistical Inference for Branching Processes. Wiley,NY.

Guttorp, P. and Perlman, M. D. (2015). Testing subcriticality vs. supercriticality in a Galton-Watson branching process with power series offspring distribution. In preparation.

Harris, T. E. (1963). The Theory of Branching Processes. Springer-Verlag, Berlin.

Heyde, C. C. (1979). On assessing the potential severity of an outbreak of a rare infectious disease: a Bayesian approach. Australian J. Statist. 21 282-292.

Jagers, P. (1975). Branching Processes with Biological Application. Wiley, NY.

Karlin, S. (1966). A First Course in Stochastic Processes. Acad. Press, NY.

Karlin, S. (1968). Total Positivity. Stanford University Press, Stanford, CA.

Kemperman, J. H. B. (1977). On the FKG-inequality for measures on a partially ordered space. Indag. Math. 39 313- 331.

Kesten, H., Ney, P., and Spitzer, F. (1966). The Galton-Watson process with mean one and finite variance. Teor. Veroyatnost. i Primenen. 11 579-611.

Lehmann, E. L. and Romano, J. P. (2005). Testing Statistical Hypotheses, Third Edition. Springer, NY.

Miller, R. S., Botkin, D. B., and Mendelssohn, R. (1974). The whooping crane (Grus americana) population of North America. Biol. Cons. 6 106-111.

Perlman, M. D. and Olkin, I. (1980). Unbiasedness of invariant tests for MANOVA and other multivariate problems. Ann. Statist. 8 1326- 1341.

Seneta, E. (1970). An explicit-limit theorem for the critical Galton-Watson process with immigration. J. Royal Statist. Soc. Series B 32 149-152.

Scott, D. (1987). On posterior asymptotic normality and asymptotic normality of estimators for the Galton-Watson process. J. Royal Statist. Soc. Series B 49 209-214. 
Stigler, S. M. (1971). The estimation of the probability of extinction and other parameters associated with branching processes. Biometrika $\mathbf{5 8}$ 499-508.

Stuart, A. and Ord, J. K. (1991). Kendall's Advanced Theory of Statistics, Vol. 2. Oxford University Press, NY.

Taylor, H. and Karlin, S. (1998). An Introduction to Stochastic Modeling, Third Edition. Academic Press, Orlando.

Waugh, W. A. (1958). Conditioned Markov processes. Biometrika 45 241-249.

Wilbur, S. R. (1978). The California Condor, 1966-76: A Look at its Past and Future. North American Fauna No. 72.136 pp. U.S. Dept. of the Interior, Fish and Wildlife Services, Washington D. C. 1978. Online at: http://library.sandiegozoo.org/journal_list/sWilbur_CAcondor.pdf. 